\title{
DEEP CHANDRA MONITORING OBSERVATIONS OF NGC 4278: CATALOG OF SOURCE PROPERTIES
}

\author{
N. J. Brassington ${ }^{1}$, G. Fabbiano ${ }^{1}$, D.-W. Kim ${ }^{1}$, A. Zezas ${ }^{1}$, S. ZepF ${ }^{2}$, A. Kundu ${ }^{2}$, L. Angelini ${ }^{3}$, R. L. Davies ${ }^{4}$, J. Gallagher ${ }^{5}$, \\ V. Kalogera ${ }^{6}$, T. Fragos $^{6}$, A. R. $\mathrm{King}^{7}$, S. Pellegrini ${ }^{8}$, And G. Trinchieri ${ }^{9}$ \\ ${ }^{1}$ Harvard-Smithsonian Center for Astrophysics, 60 Garden Street, Cambridge, MA 02138, USA; nbrassington@ head.cfa.harvard.edu \\ ${ }^{2}$ Department of Physics and Astronomy, Michigan State University, East Lansing, MI 48824-2320, USA \\ ${ }^{3}$ Laboratory for X-Ray Astrophysics, NASA Goddard Space Flight Center, Greenbelt, MD 20771, USA \\ ${ }^{4}$ Sub-Department of Astrophysics, University of Oxford, Oxford OX1 3RH, UK \\ ${ }^{5}$ Department of Astronomy, University of Wisconsin, Madison, WI 53706-1582, USA \\ ${ }^{6}$ Department of Physics and Astronomy, Northwestern University, Evanston, IL 60208, USA \\ ${ }^{7}$ Theoretical Astrophysics Group, University of Leicester, Leicester LE1 7RH, UK \\ ${ }^{8}$ Dipartimento di Astronomia, Universita di Bologna, Via Ranzani 1, 40127 Bologna, Italy \\ ${ }^{9}$ INAF-Osservatorio Astronomico di Brera, Via Brera 28, 20121 Milan, Italy \\ Received 2008 October 23; accepted 2009 February 11; published 2009 March 27
}

\begin{abstract}
We present the properties of the discrete X-ray sources detected in our monitoring program of the globular cluster (GC) rich elliptical galaxy, NGC 4278, observed with Chandra ACIS-S in six separate pointings, resulting in a co-added exposure of $458 \mathrm{ks}$. From this deep observation, 236 sources have been detected within the region overlapped by all observations, 180 of which lie within the $D_{25}$ ellipse of the galaxy. These 236 sources range in $L_{\mathrm{X}}$ from $3.5 \times 10^{36} \mathrm{erg} \mathrm{s}^{-1}$ (with $3 \sigma$ upper limit $\leqslant 1 \times 10^{37} \mathrm{erg} \mathrm{s}^{-1}$ ) to $\sim 2 \times 10^{40} \mathrm{erg} \mathrm{s}^{-1}$, including the central nuclear source which has been classified as a LINER. From optical data, $39 \mathrm{X}$-ray sources have been determined to be coincident with a GC, these sources tend to have high X-ray luminosity, with 10 of these sources exhibiting $L_{\mathrm{X}}>1 \times 10^{38} \mathrm{erg} \mathrm{s}^{-1}$. From X-ray source photometry, it has been determined that the majority of the 236 point sources that have well-constrained colors have values that are consistent with typical low-mass X-ray binary spectra, with 29 of these sources expected to be background objects from the $\log N-\log S$ relation. There are 103 sources in this population that exhibit long-term variability, indicating that they are accreting compact objects. Three of these sources have been identified as transient candidates, with a further three possible transients. Spectral variations have also been identified in the majority of the source population, where a diverse range of variability has been identified, indicating that there are many different source classes located within this galaxy.
\end{abstract}

Key words: galaxies: individual (NGC 4278) - X-rays: binaries - X-rays: galaxies

Online-only material: color figures, extended figures, machine-readable tables

\section{INTRODUCTION}

NGC 4278 is the second of two nearby, isolated, normal elliptical galaxies for which we were awarded deep monitoring observations with Chandra ACIS-S (Weisskopf et al. 2000). The purpose of these observations was to study the low-mass $\mathrm{X}$-ray binary (LMXB) population down to limiting luminosities of a few $(0.3-8.0 \mathrm{keV}) 10^{36} \mathrm{erg} \mathrm{s}^{-1}$, well in the range of those LMXBs in the Galaxy and M31. LMXBs are the only direct fossil evidence of the formation and evolution of binary stars in the old stellar populations of early-type galaxies. First discovered in the Milky Way (see Giacconi 1974), these binaries are composed of a compact accretor, neutron star or black hole, and a late-type stellar donor. The origin and evolution of Galactic LMXBs has been the subject of much discussion, centered on two main evolution paths (see Grindlay 1984; review by Verbunt \& Van den Heuvel 1995): the evolution of primordial binary systems in the stellar field, or formation and evolution in globular cluster (GC).

With the advent of Chandra, many LMXB populations have been discovered in early-type galaxies (see review Fabbiano 2006), and the same evolutionary themes (field or GC formation and evolution) have again surfaced, supported, and stimulated by a considerably larger and growing body of data. With our unique observations of NGC 3379 (Brassington et al. 2008) and NGC 4278 (this paper), we can now take a fresh look at these issues. Moreover, our observations have a time sampling that permits variability studies and the identification of X-ray transients. Such data will extend the limited sample of existing multiepoch observations (albeit with higher limiting luminosities, e.g., Irwin 2006; Sivakoff et al. 2008), where a variety of different variability behaviors of LMXBs have already been observed. Both multiepoch observations and low-luminosity thresholds are important aspects of the observational characteristics of Galactic LMXBs and are needed for constraining the evolution of these populations (e.g., Piro \& Bildsten 2002; Bildsten \& Deloye 2004).

The first galaxy that was observed in this program was NGC 3379 , in the nearby poor group Leo $(D=10.6 \mathrm{Mpc}$; Tonry et al. 2001). This system was chosen for this study because it is a relatively isolated unperturbed "typical" elliptical galaxy, with an old stellar population (age of $9.3 \mathrm{Gyr}$; Terlevich \& Forbes 2002) and a poor GC system $\left(S_{\mathrm{GC}}=1.3 \pm 0.7\right.$, Harris 1991; where $S_{\mathrm{GC}}=$ No. of GC $\left.\times 10^{\left(0.4\left(M_{V}+15\right)\right)}\right)$. From the 324 ks Chandra observation, reported in detail in Brassington et al. (2008), 132 X-ray point sources were detected, 98 of which were found to lie within the $D_{25}$ ellipse of the galaxy. Through Hubble Space Telescope (HST) observations of the galaxy, the GC system was identified in the central region and $10 \mathrm{GC}-$ LMXBs detected. These GC-LMXB associations were found to have high luminosities, with three sources exhibiting $L_{\mathrm{X}} \geqslant 1 \times$ $10^{38} \mathrm{erg} \mathrm{s}^{-1}$. Also from studying this population it was determined that $48 \%$ of the detected LMXBs were found to show some type of long-term variability, with five of these sources 
being classified as transients and a further three of these as possible transients.

The characteristics of NGC 4278 are similar to those of NGC 3379 in all but the GC population, where NGC 4278 has a rich GC system $\left(S_{\mathrm{GC}}=6.1 \pm 1.5\right.$; Harris 1991), making it an ideal galaxy to explore the evolution of LMXB from primordial field binaries in a rich environment. Like NGC 3379, NGC 4278 is a nearby $(D=16.1 \mathrm{Mpc}$; Tonry et al. 2001) relatively isolated, unperturbed "typical" elliptical galaxy with an old stellar population (age of $10.7 \mathrm{Gyr}$; Terlevich \& Forbes 2002).

Observationally, NGC 4278 is an ideal target for LMXB population studies, because of its proximity, resulting in a resolution of $\sim 36 \mathrm{pc}$ with Chandra, and its lack of a prominent hot gaseous halo. These characteristics optimize the detection of fainter LMXBs and minimize source confusion; because of its angular diameter $\left(D_{25}=4.0 \mathrm{arcmin}, \mathrm{RC} 3\right)$, NGC 4278 is entirely contained in the ACIS-S3 CCD chip, and is not affected by the degradation of the Chandra point-spread function (PSF) at large radii.

Here we publish the catalog of LMXBs with their properties resulting from the entire observational campaign of NGC 4278 (five observations between 2006 March and 2007 February, for a total of $\sim 430 \mathrm{ks}$ ), which has been recently completed and includes the first $37 \mathrm{ks}$ observation taken in 2005, from the Chandra archive. In addition to this catalog paper, further highlights from the X-ray binary population of NGC 4278 will be presented in forthcoming papers. In the first (Kim et al. 2009), we compare the X-ray luminosity function (XLF) of GC and field LMXBs detected in both NGC 4278 and NGC 3379. This will be followed by a paper presenting the properties of the transient population of both galaxies (N. J. Brassington et al. 2009, in preparation). Further papers will be presented on the properties of the nuclear source and the diffuse emission of NGC 4278, as well as the nonuniform distribution of the LMXBs within the galaxy and the intensity and spectral variability of the luminous X-ray binary population.

This paper is organized as follows: Section 2 details the observational program and describes the data analysis methods and results, including pipeline processing of the data, source detection, astrometry and matching of sources from the different observations, X-ray photometry and overall population results, and variability analysis and optical counterpart matching (GC and background objects); Section 3 describes the source catalog, including the results from the individual observations and the coadded data; Section 4 presents the discussion of the properties of the sources catalog; and Section 5 summarizes the conclusions of this work.

\section{OBSERVATIONS AND DATA ANALYSIS}

The six separate Chandra observations of NGC 4278 have been carried out over a two-year baseline, with the first of these, a 37 ks pointing, being performed in 2005 February. This observation has been followed by five deeper pointings, all carried out between 2006 March and 2007 February, resulting in a total exposure time of $471 \mathrm{ks}$.

The initial data processing to correct for the motion of the spacecraft and apply instrument calibration was carried out with the Standard Data Processing (SDP) at the Chandra XRay Center (CXC). The data products were then analyzed using the CXC CIAO software suite (ver. 3.4) ${ }^{10}$ and HEASOFT (ver. 5.3.1). The data were reprocessed, screened for bad pixels, and

\footnotetext{
10 http://asc.harvard.edu/ciao.
}

Table 1

Observation Log

\begin{tabular}{lcccc}
\hline \hline Observation Number & OBSID & Date & \multicolumn{2}{c}{ Exposure (s) } \\
\hline 1 & 4741 & 2005 Feb 3 & 37462.0 & 37264.5 \\
2 & 7077 & 2006 Mar 16 & 110303.8 & 107736.7 \\
3 & 7078 & 2006 Jul 25 & 51433.2 & 48076.2 \\
4 & 7079 & 2006 Oct 24 & 105071.7 & 102504.6 \\
5 & 7081 & 2007 Feb 20 & 110724.0 & 107564.5 \\
6 & 7080 & 2007 Apr 20 & 55824.8 & 54837.5 \\
Total & $\cdots$ & $\cdots$ & 470819.5 & 457984.0 \\
\end{tabular}

Notes. The pointing OBSID 7181 was taken before OBSID 7080, so to maintain the time sequence of the exposures these observation numbers have been labeled as above in this paper.

time filtered to remove periods of high background. Following the methods of Kim et al. (2004a), time filtering was done by making a background light curve and then excluding those time intervals beyond a $3 \sigma$ fluctuation above the mean background count rate, where the mean rate was determined iteratively after excluding the high background intervals. This resulted in a total corrected exposure time of $458 \mathrm{ks}$, the log of these exposures is presented in Table 1.

From the six individual data sets, a combined observation was produced, using the script merge_all, ${ }^{11}$ where the reprocessed level 2 event files from each observation were reprojected to a given R.A. and decl., and then combined, and a combined exposure map was also created. Each of the individual observations used in the merge_all script were first corrected for relative astrometry before being reprocessed and reprojected. These corrections were done by using the CIAO tool reproject_aspect ${ }^{12}$ to update the aspect solution files by comparing the source list from an individual observation to the source list from a single reference observation (in this case the longest cleaned exposure obs-7078). These corrected aspect solution files were then used in all subsequent analysis.

From the co-added data set of these six observations, a 0.3 $8.0 \mathrm{keV}$ (from here on referred to as "full band") Chandra image was created and adaptively smoothed using the CIAO task csmooth. This uses a smoothing kernel to preserve an approximately constant signal-to-noise ratio $(\mathrm{S} / \mathrm{N})$ across the image, which was constrained to be between $2.4 \sigma$ and $4 \sigma$. In Figure 1, both the optical image, with the full band Xray contours overlaid (top), and the "true color" image of the galaxy system (bottom) are shown. The "true color" image was created by combining three separate smoothed, and exposure corrected, images in three energy bands, $0.3-0.9 \mathrm{keV}, 0.9-2.5$ $\mathrm{keV}$, and $2.5-8.0 \mathrm{keV}$, using the same smoothing scale for each image. These energy bands correspond to red, green, and blue, respectively.

\subsection{Source Detection and Count Extraction Regions}

Discrete X-ray sources were searched for over each observation (the six single observations and the co-added observation) using the CIAO tool wavdetect, where the full band, with a significance threshold parameter of $1 \times 10^{-6}$, corresponding to roughly one spurious source over one $\mathrm{CCD}$, was searched over. This CIAO tool searches for localized enhancements of the X-ray emission, and does not set any a priori thresholds

\footnotetext{
11 http://asc.harvard.edu/ciao/ahelp/merge_all.html.

12 http://asc.harvard.edu/ciao/threads/reproject_aspect/.
} 

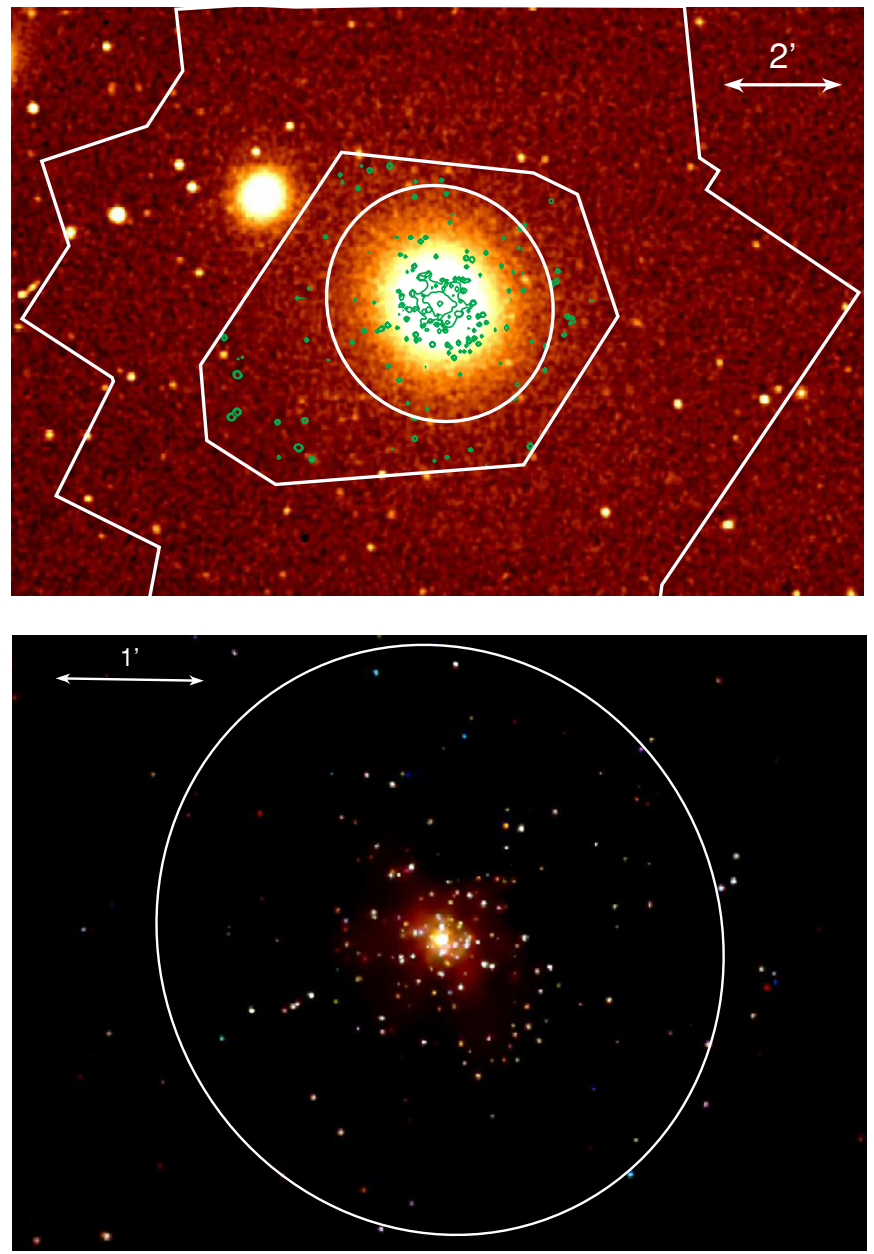

Figure 1. Top: an optical image of NGC 4278, with the outline of the total area covered by the ACIS-S3 chips, the region overlapped by all six of the pointings and the $D_{25}$ ellipse of this galaxy shown in white. Also overlaid within the overlap region are the full band adaptively smoothed, X-ray contours. Bottom: a "true color" image of the galaxy, where red corresponds to $0.3-0.9 \mathrm{keV}$, green to $0.9-2.5 \mathrm{keV}$, and blue to $2.5-8.0 \mathrm{keV}$. The $D_{25}$ ellipse of this galaxy is also shown.

on the $\mathrm{S} / \mathrm{N}$ of each source (in contrast to sliding cell algorithms; Freeman et al. 2002). In Kim et al. (2004a), simulations were carried out to investigate the number of false detections compared to the expected $\sim 1$ false source per image provided by the threshold significance of $1 \times 10^{-6}$. These simulations and results are detailed in Section 4 of the paper, where they find that the performance of wavdetect is as expected, resulting in $\sim 1$ spurious source per image. These simulations cover the values of background $\left(\sim 0.28\right.$ counts pixel $\left.{ }^{-1}\right)$ of our co-added observation. Further to this, these simulations were compared to Chandra observations with relatively long exposures $(\sim 100$ ks), where 0.3 spurious sources per exposure were detected, fully consistent with the simulation results. A similar approach was also used by Kenter et al. (2005).

Following the results of these simulations it is clear that, when setting a detection threshold of $1 \times 10^{-6}$ in wavdetect, only one of the formally identified sources is expected to be a false detection per image and so this prescription has been followed here. We reiterate that using this method does not set any a priori thresholds on the $\mathrm{S} / \mathrm{N}$ of each source, it is therefore possible to include sources that have a high detection significance but at the same time a low flux significance, or $\mathrm{S} / \mathrm{N}$, therefore resulting in sources with poorly constrained flux.
When running wavdetect a range of $1,2,4,8,16$ and 32 pixel wavelet scales were selected (where pixel width is $0 . " 49$ ), with all other parameters set at the default values. Exposure maps were created for the S3 chip from each observation, at 1.5 $\mathrm{keV}$. The wavdetect tool was used in preference to other source detection software, as this detection package can be used within the low counts regime, as it does not require a minimum number of background counts per pixel for the accurate computation of source detection thresholds. Further to this, wavdetect also performs better in confused regions, which is the case in the nuclear region of elliptical galaxies (Freeman et al. 2002).

Once the X-ray sources had been detected, and their position had been determined by wavdetect, counts were extracted from a circular region, centered on the wavdetect position, with background counts determined locally, in an annulus surrounding the source, following the prescription of Kim et al. (2004a). The extraction radius for each source was chosen to be the $95 \%$ encircled energy radius at $1.5 \mathrm{keV}$ (which varies as a function of the off-axis angle ${ }^{13}$ ), with a minimum of $3^{\prime \prime}$ near the aim point. Similarly, background counts for each source were estimated from a concentric annulus, with inner and outer radii of two and five times the source radius, respectively.

When nearby sources were found within the background region, they were excluded before measuring the background counts. Net count rates were then calculated with the effective exposure (including vignetting) for both the source and background regions. Errors on counts were derived following Gehrels (1986). For cases where sources have fewer than four counts, the Gehrels approximation begins to differ to Poissonian errors. However, these error values are still accurate to $1 \%$, and, if anything, provide a more conservative estimate as Gehrels approximation does not account for the smaller error value at the lower limit. When the source extraction regions of nearby regions were found to overlap, to avoid an overestimate of their source count rates, counts were calculated from a pie-sector, excluding the nearby source region, and then rescaled, based on the area ratio of the chosen pie to the full circular region. Once the correction factor was determined, it was applied to correct the counts in all energy bands. For a small number of sources that overlapped with nearby sources in a more complex way (e.g., overlapped with more than two sources), instead of correcting the aperture photometry, the source cell determined by wavdetect was used to extract the source counts in each energy band.

From these source counts, fluxes and luminosities were calculated in the $0.3-8.0 \mathrm{keV}$ band, with an energy conversion factor (ECF) corresponding to an assumed power-law spectral shape, with $\Gamma=1.7$ and Galactic $N_{\mathrm{H}}{ }^{14}$ (see Figure 11 for a justification of this assumption). The ECF was calculated with the $\operatorname{arf}$ (auxiliary response file) and the $r m f$ (redistribution matrix file) generated for each source in each observation. For each source, the spatial and temporal quantum efficiency variations ${ }^{15}$ were accounted for by calculating the ECF in each observation and then taking an exposure-weighted mean ECF. The ECF over the $0.3-8.0 \mathrm{keV}$ band varied by only $\sim 2 \%$ between all six of the observations taken between 2005 and $2007 .{ }^{16}$ This procedure was applied to each single observation and to the total co-added exposure.

\footnotetext{
13 See http://cxc.harvard.edu/cal/Hrma/psf/index.html.

$14 N_{\mathrm{H}}=1.76 \times 10^{20} \mathrm{~cm}^{-2}$ (from COLDEN: http://cxc.harvard.edu/toolkit/ colden.jsp).

15 See http://cxc.harvard.edu/cal/Acis/Cal_prods/qeDeg/ for the low energy QE degradation.

16 http://asc.harvard.edu/ciao/why/acisqedeg.html.
} 

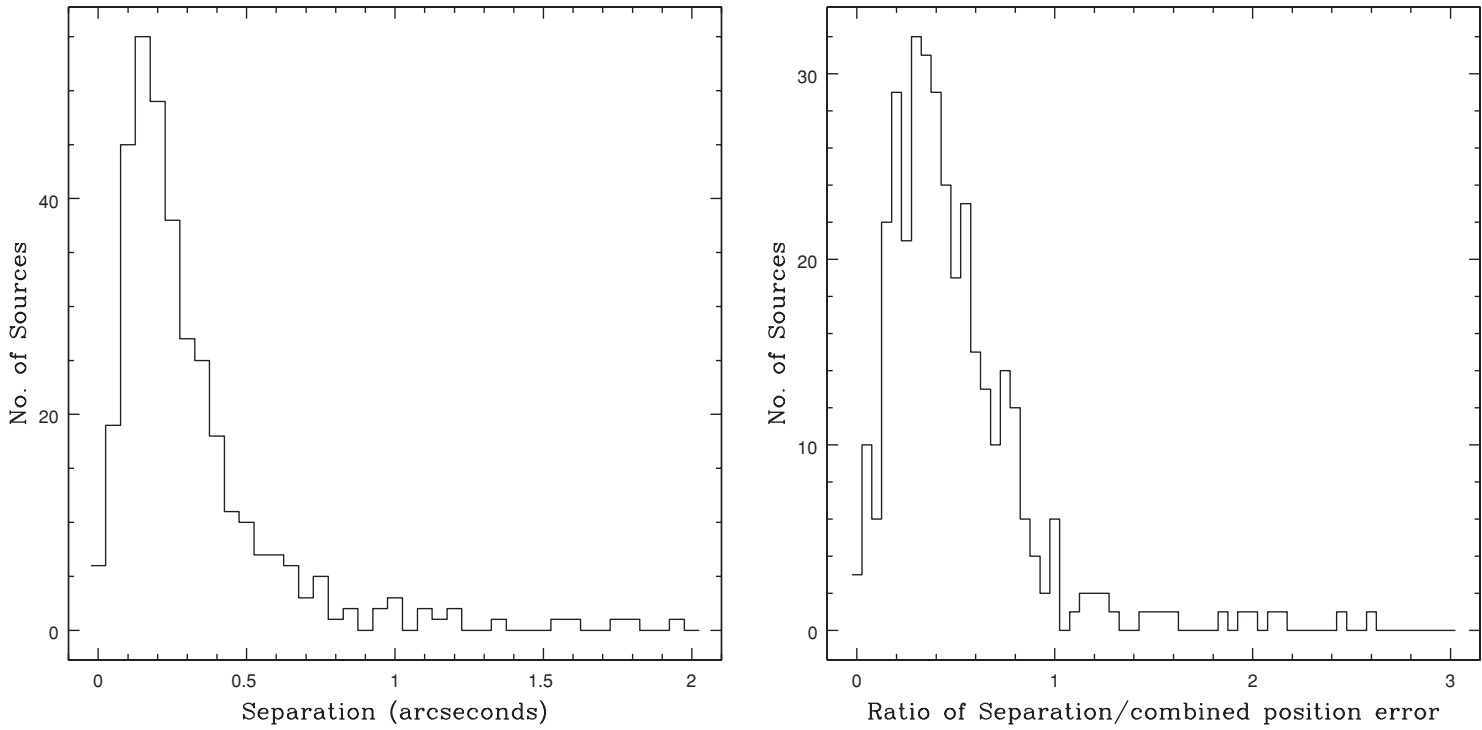

Figure 2. Left: histogram of the separation between sources detected in the co-added observation and sources detected in single observations. Right: histogram of the ratio of separation between sources detected in the co-added observation and sources detected in single observations, divided by the combined position uncertainty of these sources.

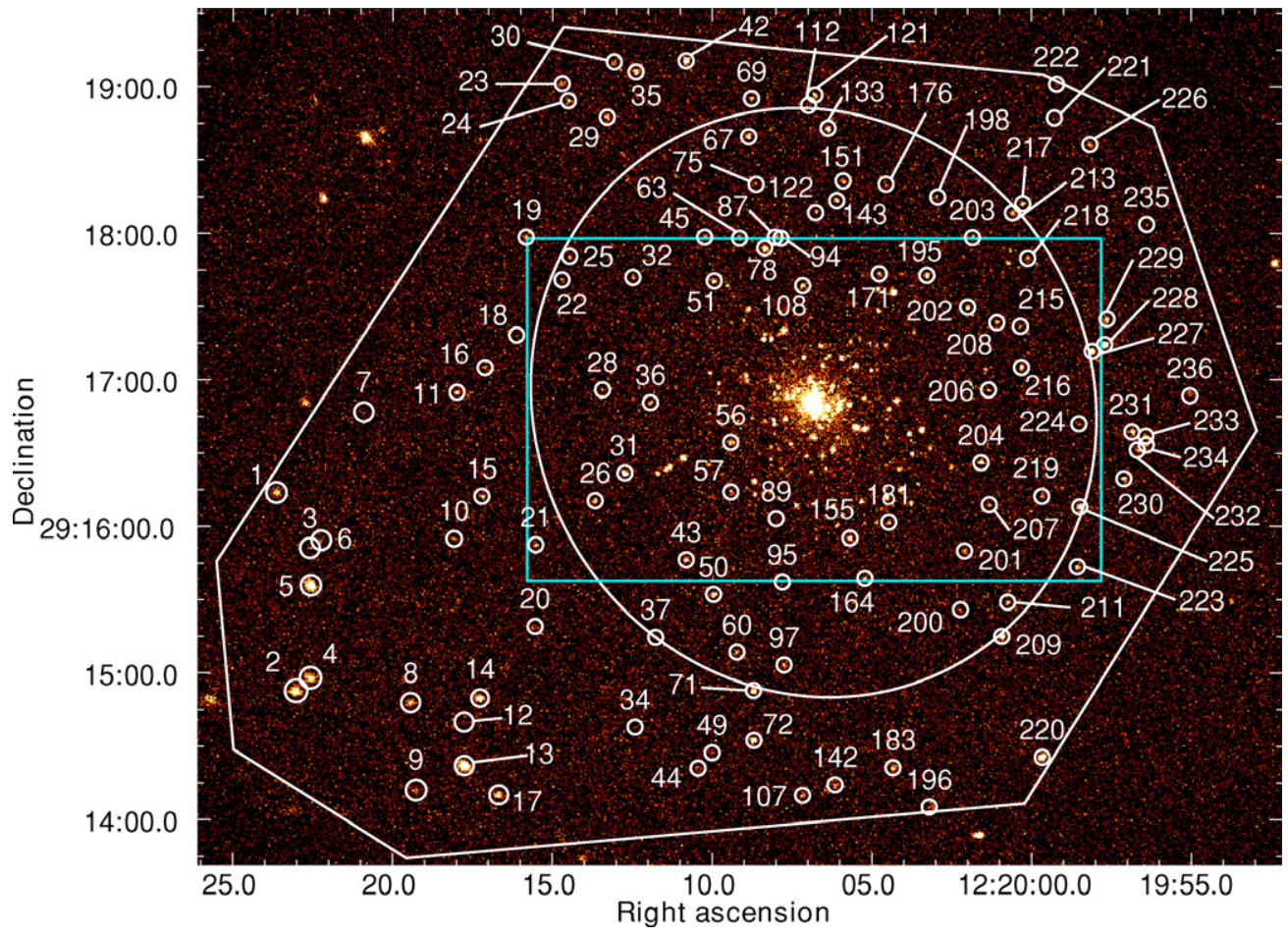

Figure 3. Main image presents a full band, raw (unsmoothed, with no exposure correction) image from the co-added observation of NGC 4278 , with the $D_{25}$ ellipse and the region overlapped by all six observations overlaid. Source region numbering corresponds to the naming convention in Table 3 and regions represent the $95 \%$ encircled energy radius at $1.5 \mathrm{keV}$. The box in the central region indicates the area shown in the next image, the central region of the galaxy, with sources labeled with the same convention as in the main image. The box shown here encloses the nuclear region of the galaxy, where there is a dense population of sources. This is presented in the final image, where these individual sources can be more clearly seen.

(A color version of this figure is available in the online journal.)

In the instances where wavdetect did not formally identify a source in a single observation, source counts have been extracted from a circle with a $95 \%$ encircled energy radius, centered on the position from the co-added observation (or in cases where the source was not formally detected in the co-added observation, the source position from the single observation was used). The definition of background regions and the treatment of overlapping sources are outlined above. From these extracted source counts, a Bayesian approach, developed by Park et al. (2006), has been used to provide $68 \%$ source intensity upper confidence bounds on the full band counts. These values have then been used to calculate upper limits on the flux and luminosity of these sources.

\subsection{Source Correlation}

From the co-added observation only, 271 sources were detected by wavdetect. From this list, sources external to the overlapping area covered by the S3 chip in all six individual 

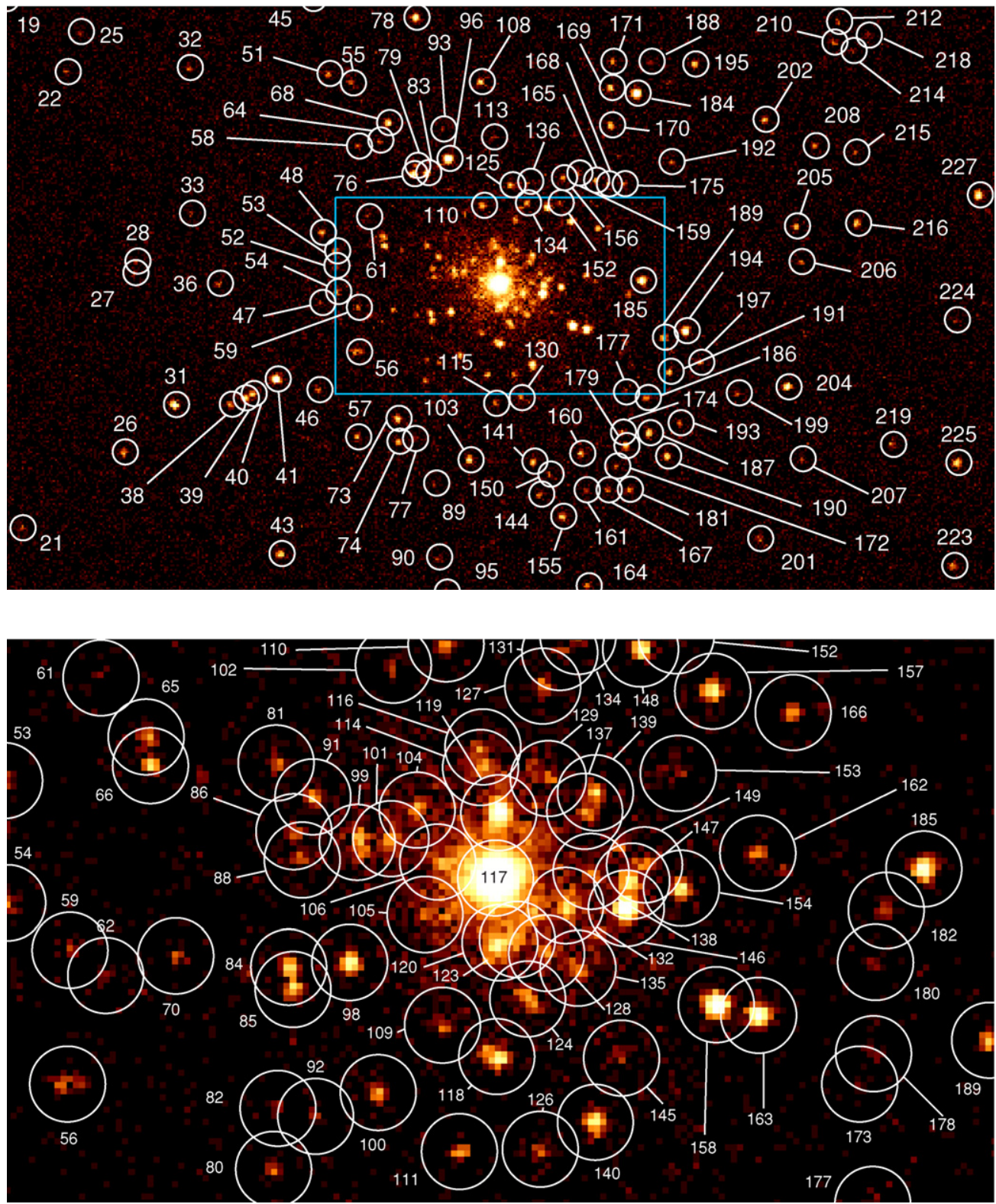

Figure 3. (Continued)

observations were excluded, reducing this total number to 220 point sources. Using this source list from the co-added observation, sources detected in the individual observations were matched with this list, where source correlations were searched for up to a separation of $2^{\prime \prime}$. In the cases where multiple matches were detected for a source, the closer correlation was selected. From these matches, a histogram of source separations, as shown in the left panel of Figure 2, was produced. In this figure it is clear that the peak separation between sources lies $\sim 0^{\prime \prime} .15$, with the number of correlated sources dropping at $\sim 1^{\prime \prime} .0$, and this is therefore the value we set for maximum separation when cross-correlating sources.

Once a cut of $1^{\prime \prime} .0$ had been applied to the cross-matched source list, the remaining unmatched sources, detected in the separate pointings only, were investigated individually, resulting in further potential matches being established. These potential matches correspond to sources with fewer counts, and hence a greater positional uncertainty, leading to larger values of separation. These potential source correlations were then further investigated by calculating the ratio of the source separation and the combined position uncertainty, where the position uncertainty at the $95 \%$ confidence level has been defined by Kim et al. (2007a) as

$$
\log \mathrm{PU}=\left\{\begin{array}{r}
0.1145 \times \text { OAA }-0.4958 \times \log C+0.1932, \\
0.0000<\log C \leqslant 2.1393, \\
0.0968 \times \text { OAA }-0.2064 \times \log C-0.4260, \\
2.1393<\log C \leqslant 3.3000
\end{array}\right.
$$

where the position uncertainty, PU, is in arcseconds, and the off-axis angle, OAA, is in arcminutes. Source counts, $C$, are as extracted by wavdetect. Using this ratio of source separation and position uncertainty allows low $L_{\mathrm{X}}$ source correlations to be identified. Often these sources, particularly at greater off-axis angles, cannot be matched by source separation cuts alone, due to the increasing PSF spread out and asymmetry at larger OAA. ${ }^{17}$ Therefore, by using this source separation-PU

\footnotetext{
${ }^{17}$ See Section 5 in Kim et al. (2004a) for a full discussion.
} 
ratio, the greater position uncertainties in these weak sources can be accounted for, resulting in smaller ratios, and thereby identifying correlations that would otherwise be missed with source separation cuts alone.

In the right panel of Figure 2, a histogram of the ratio of separation and the combined position uncertainty is shown, where sources with a ratio of greater than 1 were investigated individually. In all but one instance it was found that these higher ratio sources lie in the central region of the galaxy, where both source confusion is likely and diffuse gas may be present. This emission results in higher background fluctuations, which can lead to the PU of these sources to be underestimated, therefore resulting in a falsely high ratio value. The source that was detected outside the central region is too faint (net counts $<\sim 100$ counts) to allow its radial profiles to be compared with a corresponding model PSF profile, generated for the source position using the CIAO tool mkpsf, and has been flagged as a possible double sources.

After confirming that these additional sources with separation $\geqslant 1^{\prime \prime}$ were correlated with sources detected in the co-added observation, the remaining list of sources detected in individual observation was reduced to 16 . All of these 16 sources were determined to be well separated from the sources detected in the co-added observation, and were therefore included in the final list of detected sources, increasing the total number to 236. These 236 sources are presented in Figure 3, where the unsmoothed full band image from the co-added data set, with regions overlaid in white, is shown.

\subsection{Hardness Ratios and X-Ray Colors}

Within NGC 4278, the range of net counts for the pointlike sources in the co-added observation is $\sim 7-48772$ (with $\mathrm{S} /$ $\mathrm{N}$ values ranging from 1.1 to 218.8 ), corresponding to $0.3-$ $8.0 \mathrm{keV}$ luminosities of $3.5 \times 10^{36} \mathrm{erg} \mathrm{s}^{-1}(3 \sigma$ upper limit $\leqslant 1 \times 10^{37} \mathrm{erg} \mathrm{s}^{-1}$ ) to $2 \times 10^{40} \mathrm{erg} \mathrm{s}^{-1}$, when using the ECF described in Section 2.1. Most of these sources are too faint for detailed spectral analysis, therefore their hardness ratio and X-ray colors were calculated in order to characterize their spectral properties. The X-ray hardness ratio is defined as HR $=(\mathrm{Hc}-\mathrm{Sc}) /(\mathrm{Hc}+\mathrm{Sc})$, where $\mathrm{Sc}$ and $\mathrm{Hc}$ are the net counts in the $0.5-2.0 \mathrm{keV}$ and $2.0-8.0 \mathrm{keV}$ bands, respectively. Following the prescription of Kim et al. (2004b), the X-ray colors are defined as $\mathrm{C} 21=\log \left(\mathrm{S}_{1} / \mathrm{S}_{2}\right)$ and $\mathrm{C} 32=\log \left(\mathrm{S}_{2} / \mathrm{H}\right)$, where $\mathrm{S}_{1}$, $\mathrm{S}_{2}$, and $\mathrm{H}$ are the net counts, respectively, in the energy bands of $0.3-0.9 \mathrm{keV}, 0.9-2.5 \mathrm{keV}$, and $2.5-8.0 \mathrm{keV}$ (energy bands and definitions are summarized in Table 2). These counts were corrected for the spatial and temporal QE variation, referring them all to the aim point of the first, recent observing epoch (2006 February, Table 1), and for the effect of the Galactic absorption, using $N_{\mathrm{H}}=1.76 \times 10^{20} \mathrm{~cm}^{-2}$ (from COLDEN: http://cxc.harvard.edu/toolkit/colden.jsp).

By definition, as the X-ray spectra become harder, the HR increases and the X-ray colors decrease. For faint sources with a small number of counts, the formal calculation of the HR and colors often results in unreliable errors, because of negative net counts in one band and an asymmetric Poisson distribution. Therefore, a Bayesian approach has been applied to derive the uncertainties associated with the HR and colors. This model was developed by Park et al. (2006) and calculates values using a method based on the Bayesian estimation of the "real" source intensity, which takes into account the Poisson nature of the probability distribution of the source and background counts, as well as the effective area at the position of the source
Table 2

Definition of Energy Bands and X-Ray Colors

\begin{tabular}{ll}
\hline \hline Band & \multicolumn{1}{c}{ Definition } \\
\hline Broad $(B)$ & $0.3-8 \mathrm{keV}$ \\
Soft $(\mathrm{S})$ & $0.3-2.5 \mathrm{keV}$ \\
Hard $(\mathrm{H})$ & $2.5-8 \mathrm{keV}$ \\
Soft $1\left(\mathrm{~S}_{1}\right)$ & $0.3-0.9 \mathrm{keV}$ \\
Soft 2 $\left(\mathrm{S}_{2}\right)$ & $0.9-2.5 \mathrm{keV}$ \\
Conventional broad $(\mathrm{Bc})$ & $0.5-8 \mathrm{keV}$ \\
Conventional soft $(\mathrm{Sc})$ & $0.5-2 \mathrm{keV}$ \\
Conventional hard $(\mathrm{Hc})$ & $2-8 \mathrm{keV}$ \\
Hardness ratio HR & $(\mathrm{Hc}-\mathrm{Sc}) /(\mathrm{Hc}+\mathrm{Sc})$ \\
X-ray color C21 & $-\log \left(\mathrm{S}_{2}\right)+\log \left(\mathrm{S}_{1}\right)=\log \left(\mathrm{S}_{1} / \mathrm{S}_{2}\right)$ \\
X-ray color C32 & $-\log (\mathrm{H})+\log \left(\mathrm{S}_{2}\right)=\log \left(\mathrm{S}_{2} / \mathrm{H}\right)$ \\
\hline
\end{tabular}

(van Dyk et al. 2001), resulting in HR and color values that are more accurate than the classical method, especially in the small-number-of-counts regime (less than 10 counts), where the Poisson distributions become distinctly asymmetric.

\subsection{Source Variability}

Due to the monitoring approach that has been used when observing NGC 4278, both long-term and short-term variations have been able to be searched for in the galaxy's LMXB population. Long-term variability was defined by the chi-squared test, where a straight line model was fitted to the luminosities derived for each individual observation, with errors based on the Gehrels approximation (Gehrels 1986). For the cases where sources only had upper limit values of $L_{X}$, the associated error was defined to be the standard deviation of the upper limit from the mode value attained from the Bayesian estimate methods, resulting in a conservatively large error, due to the nature of the Poissonian statistics. From these best fit models, sources were determined to be variable if $\chi_{v}^{2}>1.2$, and those with fits with $\chi_{v}^{2}<1.2$ were defined as nonvariable sources. For sources that were only detected in the co-added observation, long-term variability was not searched for. This long-term behavior will be further investigated in a forthcoming paper, where full Poissonian error treatment will be applied to sources with very low observed counts.

In addition to the chi-squared test variability criterion, transient candidates (TCs), sources that either appear or disappear, or are only detected for a limited amount of "contiguous" time during the observations, were searched for. Typically, sources are defined to be TCs if the ratio between the "on-state," the peak $L_{X}$ luminosity, and the "off-state," the lower $L_{X}$ luminosity or nondetection upper limit, is greater than a certain value (usually between 5 and 10, e.g., Williams et al. 2008). However, such a criterion can overestimate the number of TCs, when the "on-state" X-ray luminosity is poorly constrained. To address this, the Bayesian model developed by Park et al. (2006) was used to derive the uncertainties associated with the ratio between "on-state" and "off-state." In this model, source and background counts from both the peak $L_{X}$ luminosity and the nondetection observations were used to estimate the ratio, where the differences in both the exposure and ECF values were also accounted for. From this Bayesian approach a value of peak $L_{\mathrm{X}} /$ nondetection upper limit was calculated, along with a lower bound value of this ratio. This lower bound value was then used to determine the transient nature of the source, where a ratio of greater than 10 indicated a TC and sources with a ratio between 5 and 10 were labeled as possible transient candidates (PTCs). 


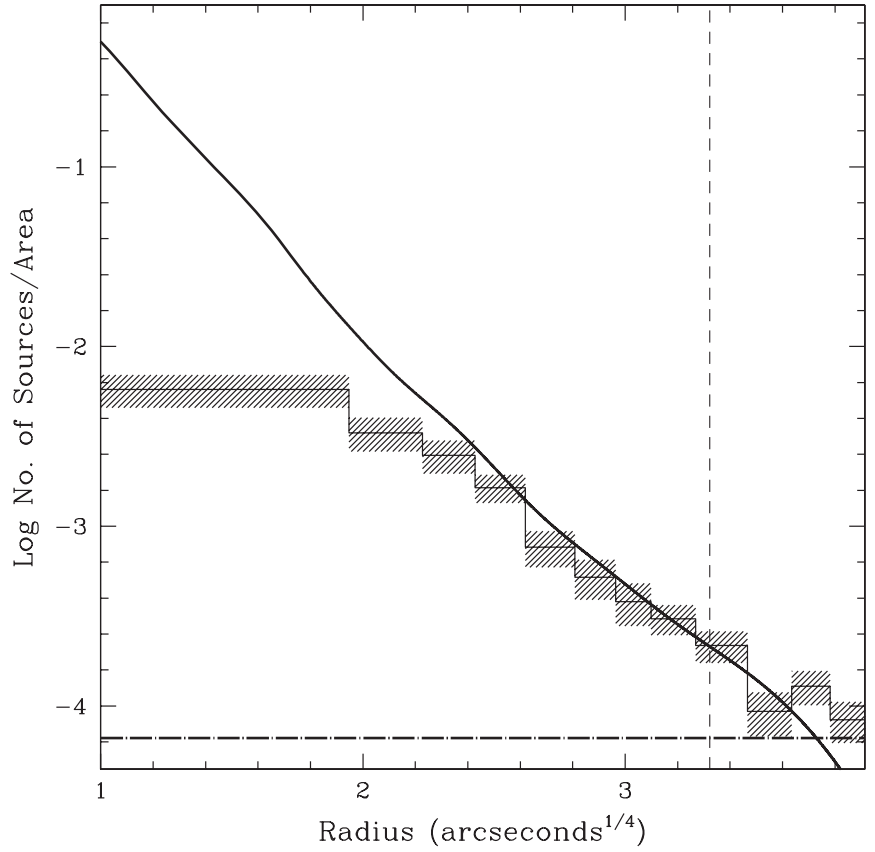

Figure 4. X-ray source density profile compared to the optical profile. The histogram indicates the $\mathrm{X}$-ray data and the thick black line is the $I$-band surface brightness best fit of Cappellari et al. (2006). The vertical dashed line is the $D_{25}$ ellipse and the horizontal dot-dashed line indicates the expected number of background sources. The central $1^{\prime \prime}$ has been excluded in this plot due to the excess optical light contribution from the LINER at the center of this galaxy.

This transient behavior was only searched for in sources that were only detected for a limited amount of "contiguous" time during the observations and were determined to be variable using the chi-squared test.

Further to these four long-term variability classifications, the variation of the source luminosity between each observation was also investigated, by comparing the significance (in $\sigma$ ) of the change in luminosity between exposures, where the significance has been estimated by

$$
\text { signif }=\frac{\left|L_{\mathrm{X} 1}-L_{\mathrm{X} 2}\right|}{\sqrt{\left(\sigma_{1}^{2}+\sigma_{2}^{2}\right)}},
$$

where $\sigma_{n}$ is the error value of the luminosity from that individual observation, based on the Gehrels approximation, or, where

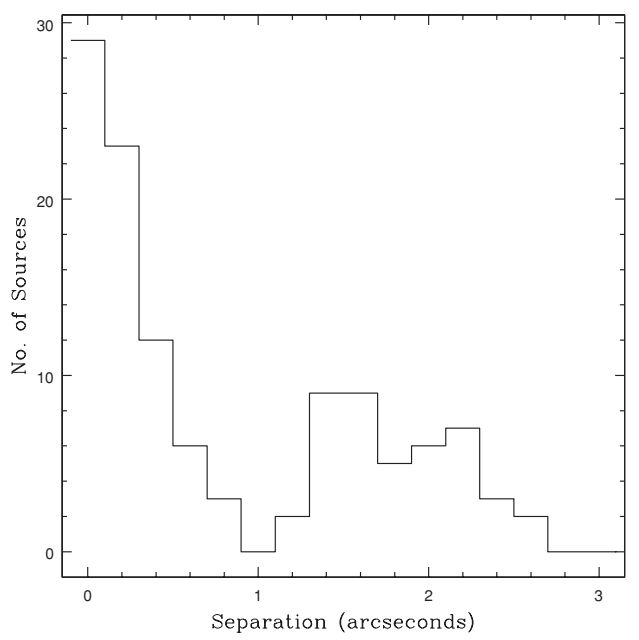

upper limits have been used, the standard deviation of the estimated luminosity.

Short-term variations in each source were investigated when net counts $>20$ in a single observation. In these instances, the variability was identified by using the Kolmogorov-Smirnov test (K-S test), where sources with variability values $>90 \%$ confidence were labeled as possible variable sources and sources with values $>99 \%$ confidence were defined as variable sources. This short-term variability was also quantified by using the Bayesian block (BB) method (Scargle 1998; J. D. Scargle et al. 2009, in preparation). This method searches for abrupt changes in the source intensity during an observation, and therefore is very efficient for detecting bursts or state changes. Because it is based on the Poisson likelihood it can be used on the unbinned light curves of sources with very few counts. The implementation of the method used in this analysis is the same as in the ChaMP pipeline (see Section 3.3.2 in Kim et al. 2004a). This assumes a prior of $\gamma=4.0$ which roughly translates to a significance level of $\sim 99 \%$ for each detected block (however see J. D. Scargle et al. 2009, in preparation, ${ }^{18}$ for a caveat on this interpretation of the value of the prior).

\subsection{Radial Profile}

From the complete source list from the co-added observation a radial distribution of LMXBs has been created, using annuli centered on the nucleus of the galaxy (source 117). This profile has been compared to a multi-Gaussian expansion model of the I-band optical data (Cappellari et al. 2006), which is assumed to follow the stellar mass of the galaxy (Gilfanov 2004). This $\mathrm{X}$-ray source density profile is presented in Figure 4, where the optical profile has been normalized to the X-ray data by way of a $\chi^{2}$ fit. The central $1^{\prime \prime}$ profile of the galaxy has not been plotted due to the excess of optical light, arising from the LINER that lies at the center of NGC 4278. Also indicated in this figure is the $D_{25}$ ellipse and the number of background sources, which has been estimated from the hard-band ChaMP+CDF $\log N-\log S$ relation (Kim et al. 2007b), where $\sim 29$ sources are expected to be objects not associated with NGC 4278. From this figure it can be seen that the X-ray profile follows the optical surface density profile at larger radii, with the flattening in the central region $\left(r \leqslant 15^{\prime \prime}\right)$, a consequence of source confusion. This indicates

${ }_{18}$ See also http://space.mit.edu/CXC/analysis/SITAR/functions.html.

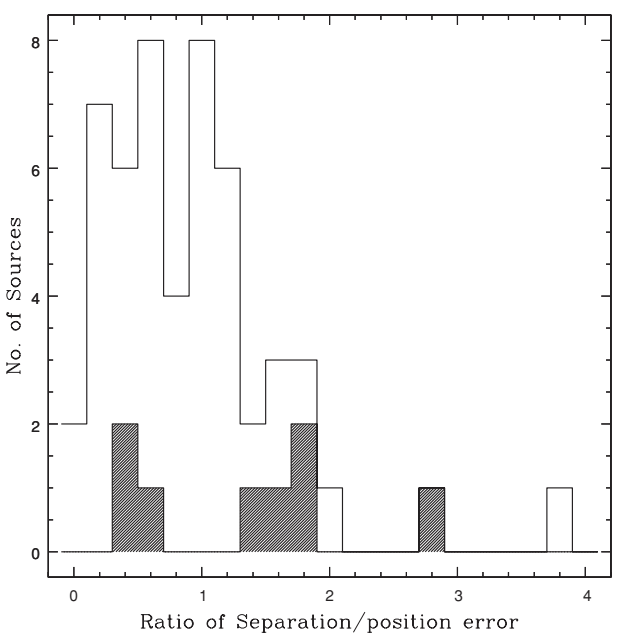

Figure 5. Left: a histogram of the separation between the co-added X-ray source position and the optical counterpart. Right: histogram of the ratio of separation divided by the position uncertainty from the X-ray point source for all optical-X-ray correlations with separations smaller than $1^{\prime \prime}$. The shaded regions indicate correlations with optical objects that have been classified as background sources (details of this classification are given in the text). 
that the number and spatial distribution of LMXBs follows that of their parent population, the old stellar population.

\subsection{Optical Counterparts}

The GC system of NGC 4278, observed with WFPC2, onboard HST, is reported in Kundu \& Whitmore (2001), where images in both the $V$ and $I$ bands have been analyzed. In addition to this GC system identified in the HST data, background objects have also been classified (A. Kundu 2007, private communication). These have been identified as objects that were well resolved in the HST images and were clearly more extended than any known GC. Further to this, these background objects often had other features, such as visible disks, indicative of a galaxy rather than a GC.

Right ascension (R.A.) and declination (decl.) corrections have been applied to the astrometry of the HST data, relative to the co-added Chandra observation. This was done by comparing the positions of all GC-LMXB correlations with separations $\leqslant 1^{\prime \prime}$ and with net counts $\geqslant 50$, resulting in 37 matches. From these matches relative offsets of $\Delta$ R.A. $=0 . " 48$ and $\Delta$ decl. $=$ $0 . " 22$ were identified and removed from the HST data.

After correcting the astrometry of these optical data, correlations up to an offset of $3^{\prime \prime}$ with the X-ray sources were searched for. When multiple matches were found, the closer matching object was selected. In the left panel of Figure 5, a histogram of these matches is shown, where it can be seen that the number of source correlations decreases at $\sim 1^{\prime \prime}$ before increasing at greater separations. Because of this clear distinction, the source radius was set to $1^{\prime \prime}$ and sources between $1^{\prime \prime}$ and $3^{\prime \prime}$ were defined as "excluded matches." This cutoff radius value was then tested by comparing these correlations with the ratio of the separation divided by the combined position uncertainty from the co-added $\mathrm{X}$-ray point sources (the definition of this is given in Equation (1)) and the uncertainty in the astrometry in the optical data, which has been conservatively set at $0 .{ }^{\prime \prime} 2$.

These ratios are shown in the right panel in Figure 5, where a histogram of all optical-X-ray correlations is presented, with a shaded histogram of the background correlations only overlaid. From this figure, it is shown that the majority of the confirmed GC correlated sources have a separation-position uncertainty ratio of less than 1.4. All of the sources with a ratio of greater than 1.4 were visually inspected and only two sources are found to have small separations $<0 . " 5$. The remaining sources all have separations $>0$. ." 6 and have been identified as excluded matches. From this further analysis, the separation value cutoff was redefined to be $0 . " 6$. This results in $45 \mathrm{X}$-ray-optical correlations, six of which have been classified as background objects, which is consistent with the number of sources that are expected to be background objects from the $\log N-\log S$ relation (5). This leaves $39 \mathrm{GC}-\mathrm{X}$-ray sources that are classified as correlations. The optical properties of these GC-LMXB sources and the "excluded matches" are shown in Tables 11 and 12, respectively (full descriptions of these tables are given in Section 3).

In order to estimate the chance coincidence probability of the sources within the HST field of view (FOV), the same method as in Zezas et al. (2002) was followed, where the positions of the GCs were randomized by adding a random shift between $0 .{ }^{\prime \prime} 6$ and $30^{\prime \prime}$, and for each new fake data set the cross-correlation was performed using the same search radius as for the observed list of GCs. The limits of the shifts were chosen so that the new positions did not fall within the search radius and that they follow the general spatial distribution of the GCs. 500 such simulations
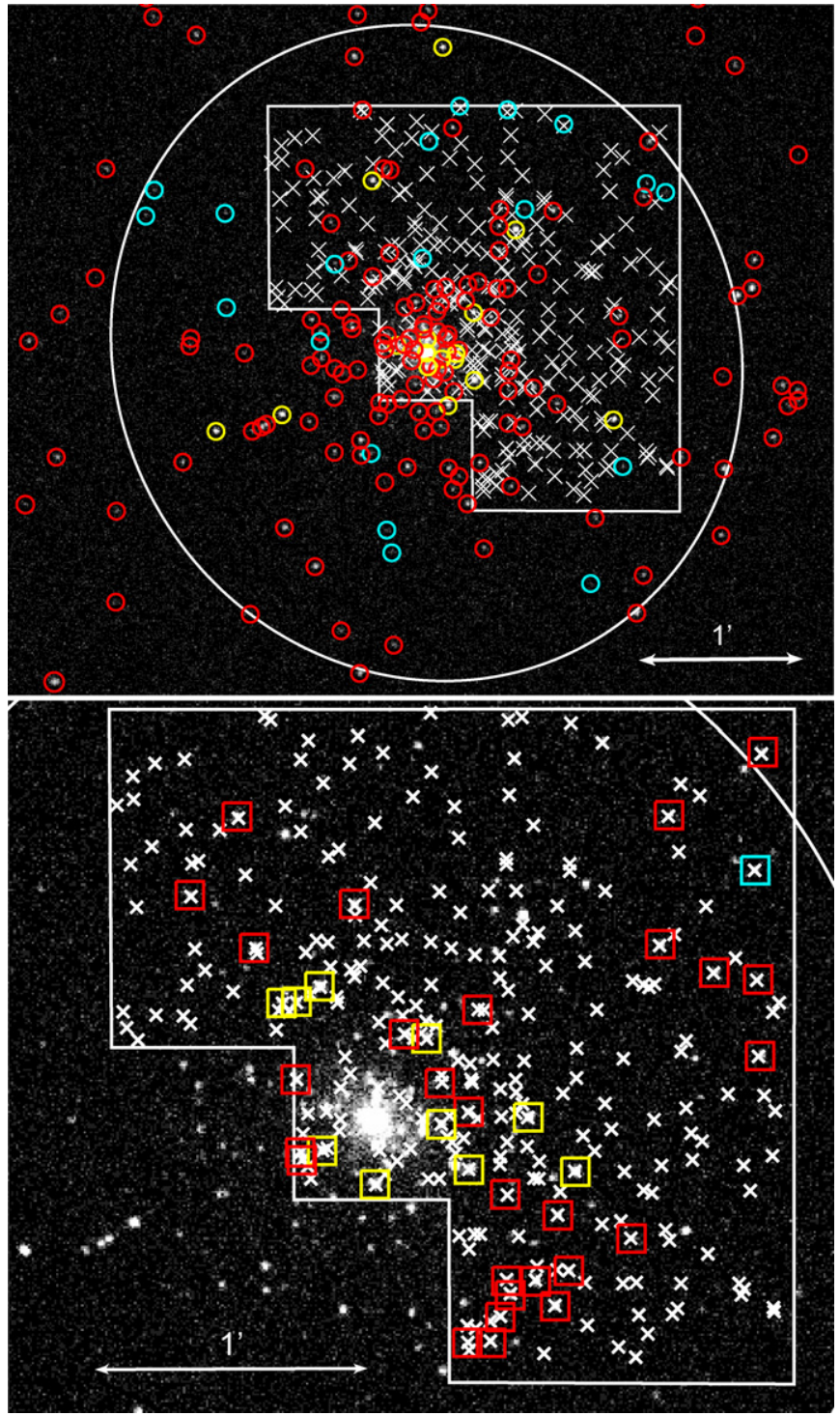

Figure 6. Full band X-ray images from the co-added observation of NGC 4278. Both images show confirmed GCs from the HST observation indicated by white "X" marks. The top image shows all X-ray sources not correlated with a GC and the bottom image indicates all correlated X-ray sources. Region colors indicate the $0.3-8.0 \mathrm{keV}$ luminosity of the source from the co-added observation: yellow regions indicate $L_{\mathrm{X}} \geqslant 1 \times 10^{38} \mathrm{erg} \mathrm{s}^{-1}$, red regions have $1 \times 10^{38} \geqslant L_{X} \geqslant 1 \times 10^{37} \mathrm{erg} \mathrm{s}^{-1}$, and cyan regions show sources with $L_{\mathrm{X}} \leqslant 1 \times 10^{37} \mathrm{erg} \mathrm{s}^{-1}$. Also shown in white are the $D_{25}$ ellipse and the HSTFOV.

were performed, resulting in $2.05 \pm 1.43$ associations expected by chance. If the cross-correlation radius is increased to $1^{\prime \prime}$, the chance associations rise to $4.96 \pm 2.10$. Increasing this radius to $3^{\prime \prime}$ results in $35.85 \pm 6.00$ associations expected by chance, which is slightly higher than the 31 "excluded matches" that have been found within this radius.

In Figure 6 the confirmed GC and X-ray sources, as well associated correlations, are indicated. In the top image, circular regions indicating the X-ray sources with no GC counterpart are overlaid on a full band X-ray image, where the confirmed GCs are indicated by white " $X$ " marks. Also in this image the $D_{25}$ ellipse and HST FOV are also shown. In the bottom image a full band X-ray image covering the HST FOV is presented, where the correlated $\mathrm{X}$-ray sources are indicated by box regions and the white " $X$ " marks indicate the confirmed GCs. In both images X-ray luminosities are indicated by color, where sources with $L_{\mathrm{X}} \geqslant 1 \times 10^{38} \mathrm{erg} \mathrm{s}^{-1}$ are shown in yellow, sources with 
Table 3

Master Source List

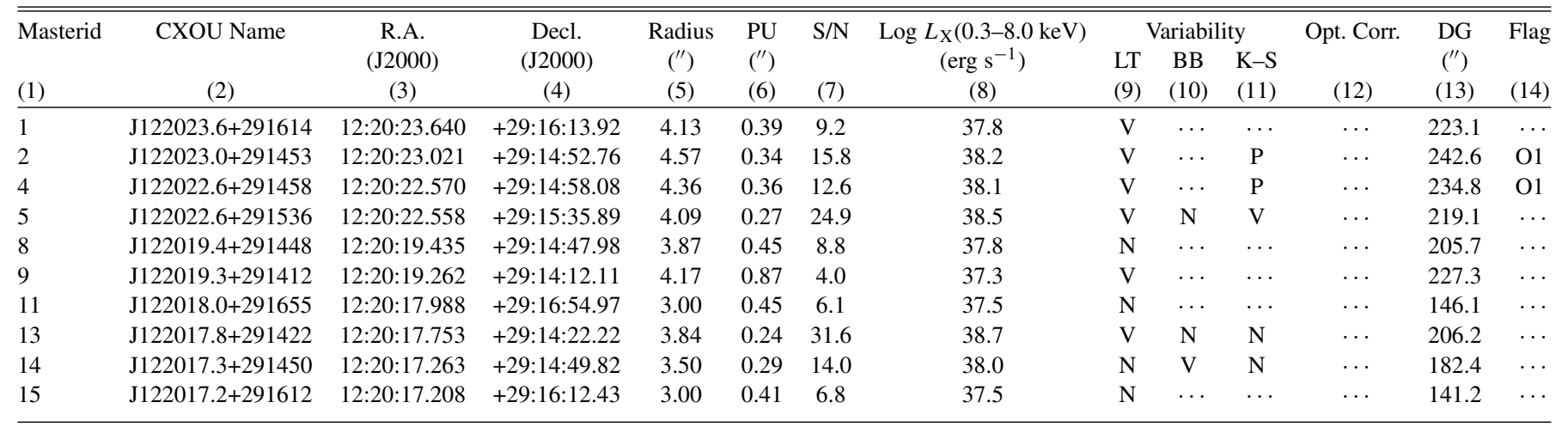

Notes. Table has been divided into two parts. The first part lists all sources with $\mathrm{S} / \mathrm{N}>3$, in at least one observation. The second section lists the remaining sources that have $\mathrm{S} / \mathrm{N}<3$ in all observations. Column 1: source number; Column 2: IAU name (following the convention "CXOU Jhhmmss.s+/-ddmmss"); Columns 3 and 4: right ascension and declination; Column 5: source extraction radius in arcseconds; Column 6: position uncertainty (from Section 2.2, Equation (1)); Column 7: signal-to-noise ratio; Column 8: $\log L_{\mathrm{X}}(0.3-8.0 \mathrm{keV})$ assuming $D=16.1 \mathrm{Mpc}$ (for the sources with $\mathrm{S} / \mathrm{N}<3,3 \sigma$ upper limits are also quoted in brackets). For sources only detected in a single observation, $1 \sigma$ upper limits from the co-added observation are quoted (with $3 \sigma$ upper limits from the detected observation presented in brackets); Column 9: long-term source variability-(N) indicates nonvariable sources, (V) indicates variable sources, (TC) transient candidates, (PTC) possible transients; Columns 10 and 11: short-term variability, where (BB) indicates Bayesian block analysis and (K-S) indicates the Kolmogorov-Smirnov test, in both columns $(\mathrm{N})$ indicates nonvariable in all observations, $(\mathrm{V})$ variable in at least one observation, and $(\mathrm{P})$ possible variability in at least one observation; Column 12: optical associations- "GC" indicates that the optical source is a confirmed globular cluster, "BG" indicates that optical source is a background objects, "corr" denotes matches that have been defined as correlations, "exmt" denotes matches between 0" 6 and 3" in separation, and "none" indicates sources inside the field of view of the HST observation, but have no optical counterpart. All other sources are external to the HST FOV. Column 13: distance from the galactic center (in arcseconds), where values in boldface indicate sources that lie within the $D_{25}$ ellipse. Column 14: flag information—(X) sources detected in a single observation only, $\mathrm{O} 1$ and $\mathrm{O} 2$ overlapping sources (single and complicated cases, respectively) and (double?) possible double sources.

(This table is available in its entirety in a machine-readable form in the online journal. A portion is shown here for guidance regarding its form and content.)

$1 \times 10^{38} \geqslant L_{\mathrm{X}} \geqslant 1 \times 10^{37} \mathrm{erg} \mathrm{s}^{-1}$ are shown in red, and sources with $L_{\mathrm{X}} \leqslant 1 \times 10^{37} \mathrm{erg} \mathrm{s}^{-1}$ are indicated in cyan.

\section{SOURCE CATALOG AND VARIABILITY ATLAS}

Table 3 presents the properties of the master list of the 236 X-ray sources detected within NGC 4278 , from the co-added observation of $458 \mathrm{ks}$. This table has been divided into two sections, where the first part presents all sources with $\mathrm{S} / \mathrm{N}>3$ in at least one observation, and the second part lists all sources with $\mathrm{S} / \mathrm{N}<3$. In this table Column 1 gives the source number used through out this series of papers, Column 2 gives the IAU name (following the convention "CXOU Jhhmmss.s+/-ddmmss"), Columns 3 and 4 give the R.A. and decl. of the source aperture, Columns 5 and 6 give the radius and the position uncertainty (PU) of the source (both in arcseconds), Column 7 gives the $\mathrm{S} / \mathrm{N}$, Column 8 gives the log value of the co-added luminosity in the $0.3-8.0 \mathrm{keV}$ energy band (for sources with $\mathrm{S} / \mathrm{N}<3,3 \sigma$ upper limit values are also presented in brackets). For sources detected in a single observation only, $1 \sigma$ upper limits from the co-added observation are shown, with $3 \sigma$ upper limit values from the detected observation presented in brackets. Column 9 provides information about the long-term variability of the source, indicating if the source is nonvariable $(\mathrm{N})$, variable (V), a TC or a possible transient (PTC). In all other cases the source was only detected in the combined observation, providing insufficient information to investigate long-term variability. In Columns 10 and 11 , the short-term variability of the source is indicated from both Bayesian block (BB) analysis and the Kolmogorov-Smirnov (K-S) test, where "V" indicates that the source is variable in at least one observation and " $\mathrm{N}$ " indicates that it has been found to be nonvariable in all six observations. In the $\mathrm{K}-\mathrm{S}$ column, sources have also been labeled as possible variable sources $(\mathrm{P})$ (see Section 2.4 for further information).
In all other cases, there were insufficient counts to investigate the short-term variability. In Column 12 the optical associations with the X-ray source are indicated, where "GC" indicates that the associated optical sources have been confirmed as a GC, and "BG" indicates that the sources have been classified as a background object. "corr" denotes matches that have been defined as correlations, and "exmt" denotes the "excluded matches," between 0'.6 and 3" in separation. Sources with a "none" label were inside the FOV of the HST observation, but have no optical counterpart. All other sources were external to the HST FOV. Column 13 gives the distance from the galactic center (in arcseconds), where values in boldface indicate sources that lie within the $D_{25}$ ellipse. Column 14 provides source flag information, indicating sources that have been detected in a single observation only (X), overlapping sources (O1 for single overlaps and $\mathrm{O} 2$ for more complicated cases) and possible double sources (double?).

In this table, the 236 sources presented are the complete list detected by wavdetect, for which we estimate that $\sim 1$ source is a spurious detection (see Section 2.1). Since this catalog of X-ray sources is intended to be as complete a study as possible, all detected sources are included in the complete list, although for sources with $\mathrm{S} / \mathrm{N}<3$ source parameters such as flux, hardness ratio, and color values are not as well constrained as sources with higher flux significance. We have therefore separated the table into two sections, where the first part presents sources with $\mathrm{S} / \mathrm{N}$ $>3$ in at least one observation and well-constrained properties, and the second part lists the sources with low-S/N values.

Table 4 presents the detailed source parameters from the coadded observation: Column 1 gives the source number, Columns 2-8 give the net counts, in each of the seven energy bands (see Table 2 for definitions of these bands), Column 9 indicates the hardness ratio, Columns 10 and 11 show the color-color values, and Column 12 gives the $\log$ value of the luminosity in the 

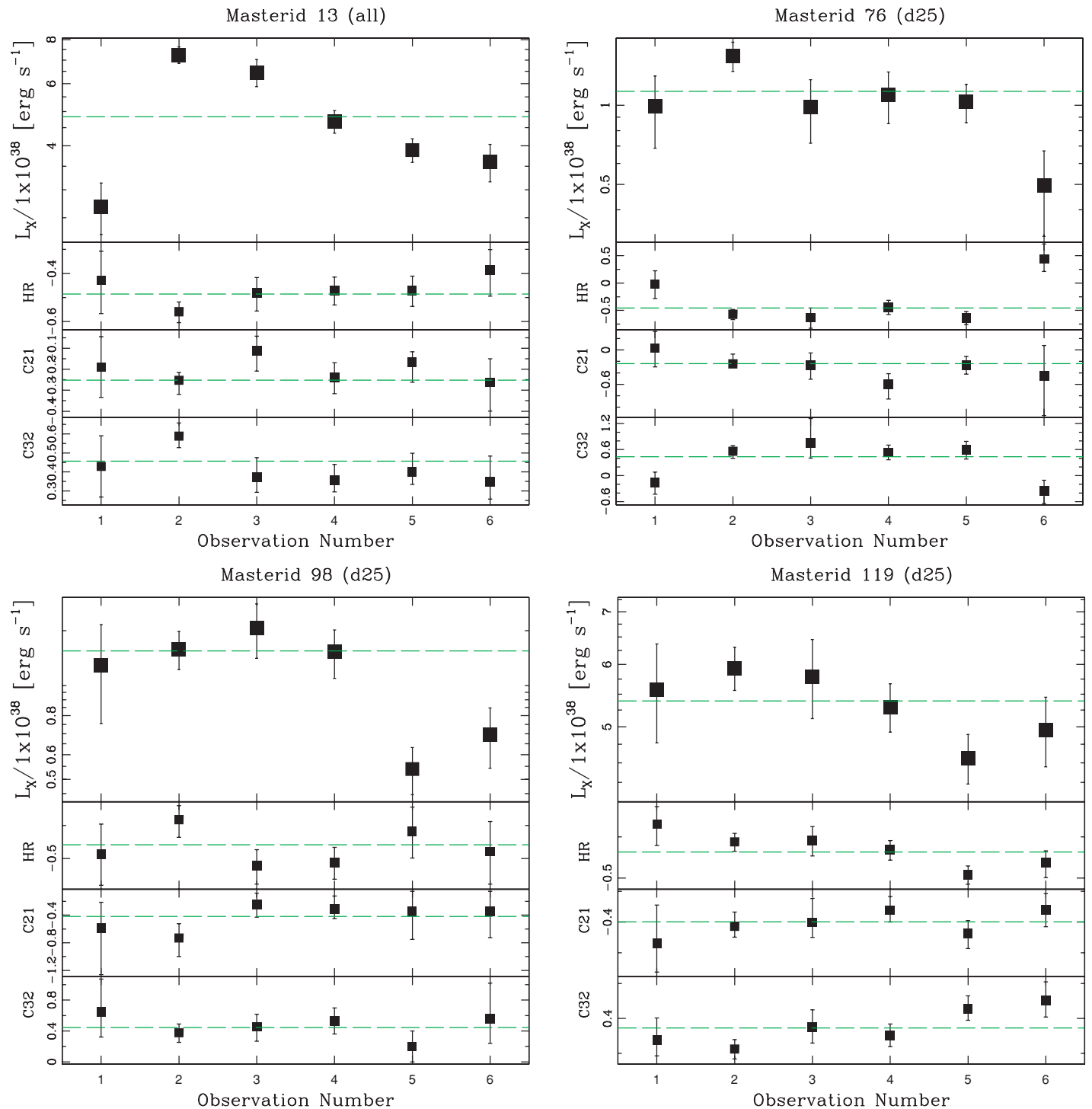

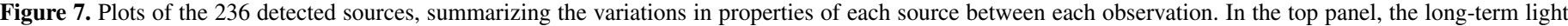

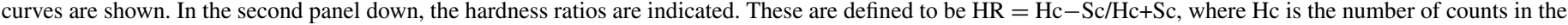

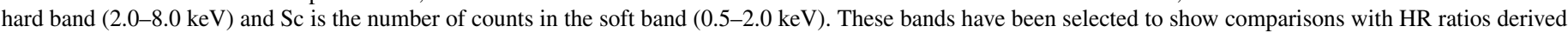

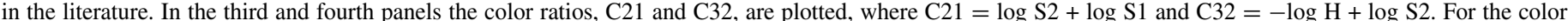

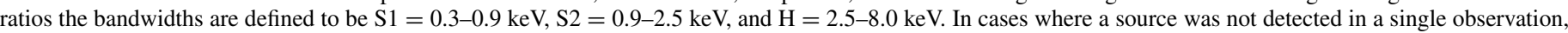

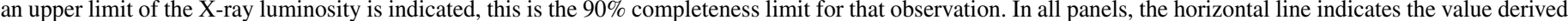
from the co-added observation.

(An extended and color version of this figure is available in the online journal.)

0.3-8.0 keV energy band. Where sources were not detected in the co-added observation, upper limits for net broadband counts and $L_{\mathrm{X}}$ are given.

Tables 5-10 present the source parameters, measured for each observation, where Columns 1-11 provide the same information presented in Table 4, but further provided in this table is source variability information, where Columns 12-14 present results of Bayesian block (BB) analysis, the Kolmogorov-Smirnov (KS) test and the significance of the change in $L_{X}$ between the previous observation and the current observation, respectively. Column 15 indicates the log value of the luminosity in the $0.3-$ $8.0 \mathrm{keV}$ energy band.

Table 11 presents the optical properties of the counterparts found from the optical data of NGC 4278, where 39 GCs and six background objects have been found to be coincident with $\mathrm{X}$ ray sources. Table 12 summarizes the results for the "excluded matches" sources. In both tables, Column 1 gives the X-ray source number, Column 2 the $V$-band magnitude, Column 3 the $I$-band magnitude, Column $4 V-I$ colors, Column 5 the separation between the X-ray source and the GC, and Column 6 the ratio between separation and the combined position error. The horizontal line in both tables separates the confirmed GCs (the top section of table) from the background objects (the bottom section of table).

Figure 7 presents the intensity and spectral variability of each of the $236 \mathrm{X}$-ray sources, over all six pointings, where the temporal properties of each point source are shown in four separate panels. In the top panel the long-term light curve of each source is presented, with error bars indicating the $1 \sigma$ uncertainty in the intensity of the source, with upper limit values provided for sources that were not detected in a single observation. The second panel shows the hardness ratio variation of each source, and panels three and four show the temporal properties of $\mathrm{C} 21$ and $\mathrm{C} 32$, respectively. In all four panels, the co-added values are 
Table 4

Source Counts, Hardness Ratios, and Color-Color Values: Co-Added Observation

\begin{tabular}{|c|c|c|c|c|c|c|c|c|c|c|c|}
\hline $\begin{array}{l}\text { Master ID } \\
\text { (1) }\end{array}$ & $\begin{array}{l}\text { B-Band } \\
\text { (2) }\end{array}$ & $\begin{array}{l}\text { S1-Band } \\
\text { (3) }\end{array}$ & $\begin{array}{c}\text { S2-Band } \\
\text { (4) }\end{array}$ & $\begin{array}{l}\text { Net Counts } \\
\text { S-Band } \\
\text { (5) }\end{array}$ & $\begin{array}{c}\text { H-Band } \\
(6)\end{array}$ & $\begin{array}{c}\text { Sc-Band } \\
(7)\end{array}$ & $\begin{array}{c}\text { Hc-Band } \\
(8)\end{array}$ & $\begin{array}{l}\text { HR } \\
(9) \\
\end{array}$ & $\begin{array}{l}\mathrm{C} 21 \\
(10) \\
\end{array}$ & $\begin{array}{l}\text { C32 } \\
\text { (11) } \\
\end{array}$ & $\begin{array}{c}\log L_{X} \\
(0.3-8.0 \mathrm{keV}) \\
(12) \\
\end{array}$ \\
\hline 1 & $139.8 \pm 15.2$ & $-2.3 \pm 4.7$ & $48.0 \pm 8.9$ & $45.6 \pm 9.7$ & $94.2 \pm 12.2$ & $39.1 \pm 8.4$ & $103.4 \pm 12.7$ & $0.40_{-0.09}^{+0.09}$ & $-1.42_{-0.80}^{+0.60}$ & $-0.26_{-0.09}^{+0.09}$ & 37.8 \\
\hline 2 & $386.2 \pm 24.4$ & $57.5 \pm 9.6$ & $237.4 \pm 17.0$ & $294.9 \pm 19.1$ & $91.8 \pm 12.4$ & $258.6 \pm 17.7$ & $117.5 \pm 13.7$ & $-0.42_{-0.06}^{+0.04}$ & $-0.54_{-0.07}^{+0.08}$ & $0.44_{-0.07}^{+0.05}$ & 38.2 \\
\hline 3 & $7.8 \pm 11.2$ & $-0.8 \pm 5.0$ & $9.5 \pm 6.1$ & $8.7 \pm 7.4$ & $-1.6 \pm 6.5$ & $10.3 \pm 6.5$ & $-2.3 \pm 6.7$ & $-0.75_{-0.25}^{+0.22}$ & $-0.49_{-1.02}^{+0.57}$ & $0.63_{-0.72}^{+0.86}$ & 36.5 \\
\hline 4 & $266.1 \pm 21.1$ & $78.8 \pm 10.7$ & $145.3 \pm 13.8$ & $224.1 \pm 17.0$ & $42.2 \pm 9.8$ & $195.7 \pm 15.7$ & $58.1 \pm 10.9$ & $-0.58_{-0.06}^{+0.06}$ & $-0.17_{-0.06}^{-0.06}$ & $0.55^{+0.11}$ & 38.1 \\
\hline 5 & $725.7 \pm 29.1$ & $202.2 \pm 15.8$ & $398.3 \pm 21.3$ & $600.5 \pm 26.1$ & $125.2 \pm 13.6$ & $508.6 \pm 24.0$ & $174.7 \pm 15.5$ & $\begin{array}{r}-0.06 \\
-0.53_{-0.03}^{+0.03}\end{array}$ & $-0.21_{-0.04}^{+0.03}$ & $0.52_{-0.04}^{-0.08}$ & 38.5 \\
\hline 6 & $17.5 \pm 11.7$ & $-6.7 \pm 4.1$ & $9.1 \pm 5.9$ & $2.5 \pm 6.7$ & $14.4 \pm 7.6$ & $4.7 \pm 5.8$ & $17.9 \pm 8.1$ & $0.59_{-0.19}^{+0.41}$ & $\begin{array}{r}-0.04 \\
-0.90_{-0.95}^{+0.97}\end{array}$ & $-0.21_{-0.36}^{+0.45}$ & 36.9 \\
\hline 7 & $\leqslant 10.8$ & $\ldots$ & $\ldots$ & $\ldots$ & & & & $\ldots$ & & & $\leqslant 36.7$ \\
\hline 8 & $128.6 \pm 14.5$ & $32.9 \pm 7.7$ & $76.6 \pm 10.5$ & $109.6 \pm 12.6$ & $19.0 \pm 7.9$ & $93.8 \pm 11.4$ & $30.1 \pm 8.9$ & $-0.56_{-0.11}^{+0.09}$ & $-0.28_{-0.10}^{+0.11}$ & $0.63_{-0.19}^{+0.18}$ & 37.7 \\
\hline 9 & $46.7 \pm 11.6$ & $16.4 \pm 6.6$ & $23.6 \pm 7.3$ & $40.0 \pm 9.4$ & $6.7 \pm 7.2$ & $34.9 \pm 8.3$ & $9.4 \pm 7.8$ & $-0.67_{-0.33}^{+0.11}$ & $-0.09_{-0.18}^{+0.22}$ & $0.53_{-0.44}^{+0.53}$ & 37.3 \\
\hline 10 & $7.7 \pm 7.8$ & $4.9 \pm 4.7$ & $5.1 \pm 5.0$ & $10.1 \pm 6.4$ & $-2.4 \pm 5.1$ & $9.4 \pm 5.7$ & $-2.7 \pm 5.3$ & $-0.80_{-0.20}^{+0.19}$ & $0.15_{-0.72}^{+0.61}$ & $0.34_{-0.64}^{-0.44}$ & 36.5 \\
\hline
\end{tabular}

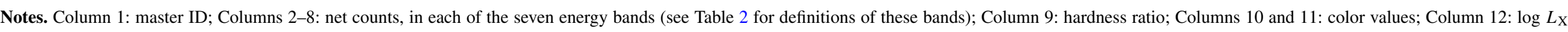
(0.3-8.0 keV). Upper limit $L_{\mathrm{X}}$ values are at the $68 \%$ confidence level.

(This table is available in its entirety in a machine-readable form in the online journal. A portion is shown here for guidance regarding its form and content.)

Table 5

Source Counts, Hardness Ratios, Color-Color Values, and Variability: Observation 1

\begin{tabular}{|c|c|c|c|c|c|c|c|c|c|c|c|c|c|c|}
\hline \multirow{2}{*}{$\begin{array}{l}\text { Master ID } \\
\text { (1) }\end{array}$} & \multicolumn{7}{|c|}{ Net Counts } & \multirow{2}{*}{$\begin{array}{l}\text { HR } \\
\text { (9) }\end{array}$} & \multirow{2}{*}{$\begin{array}{l}\mathrm{C} 21 \\
(10)\end{array}$} & \multirow{2}{*}{$\begin{array}{l}\text { C32 } \\
\text { (11) }\end{array}$} & \multicolumn{3}{|c|}{ Variability } & \multirow{2}{*}{$\begin{array}{c}\log L_{\mathrm{X}} \\
(0.3-8.0 \mathrm{keV}) \\
(15)\end{array}$} \\
\hline & $\begin{array}{l}\text { B-Band } \\
\text { (2) }\end{array}$ & $\begin{array}{l}\text { S1-Band } \\
\text { (3) }\end{array}$ & $\begin{array}{l}\text { S2-Band } \\
\text { (4) }\end{array}$ & $\begin{array}{c}\text { S-Band } \\
\text { (5) }\end{array}$ & $\begin{array}{l}\text { H-Band } \\
\text { (6) }\end{array}$ & $\begin{array}{l}\text { Sc-Band } \\
(7)\end{array}$ & $\begin{array}{c}\text { Hc-Band } \\
(8)\end{array}$ & & & & $\begin{array}{l}\text { BB } \\
\text { (12) }\end{array}$ & $\begin{array}{l}\mathrm{K}-\mathrm{S} \\
(13)\end{array}$ & $\begin{array}{c}\text { Significance } \\
\text { (14) }\end{array}$ & \\
\hline 1 & $9.8 \pm 4.7$ & $-1.0 \pm 1.9$ & $1.1 \pm 2.7$ & $0.1 \pm 2.7$ & $9.7 \pm 4.4$ & $0.0 \pm 2.3$ & $10.5 \pm 4.6$ & $0.94_{-0.08}^{+0.06}$ & $-0.38_{-115}^{+0.91}$ & $-0.72_{-0.67}^{+0.43}$ & $\ldots$ & $\ldots$ & $\ldots$ & 37.7 \\
\hline 2 & $11.3 \pm 5.0$ & $3.0 \pm 3.2$ & $5.1 \pm 3.6$ & $8.0 \pm 4.3$ & $3.3 \pm 3.4$ & $7.9 \pm 4.1$ & $3.0 \pm 3.4$ & $-0.58_{-0.28}^{+0.24}$ & $-0.08_{-0.43}^{+0.35}$ & $0.19_{-0.38}^{+0.45}$ & $\ldots$ & $\ldots$ & $\ldots$ & 37.8 \\
\hline 3 & $\leqslant 3.9$ & & $\ldots$ & $\ldots$ & & $\ldots$ & $\ldots$ & & -0.43 & & $\ldots$ & $\ldots$ & $\ldots$ & $\leqslant 37.3$ \\
\hline 4 & $15.5 \pm 5.5$ & $6.2 \pm 3.8$ & $9.1 \pm 4.3$ & $15.3 \pm 5.2$ & $0.3 \pm 2.7$ & $12.9 \pm 4.8$ & $2.1 \pm 3.2$ & $-0.81_{-0.19}^{+0.11}$ & $-0.05_{-0.24}^{+0.24}$ & $0.84_{-0.46}^{+0.84}$ & $\ldots$ & $\ldots$ & $\ldots$ & 37.9 \\
\hline 5 & $64.3 \pm 9.3$ & $19.8 \pm 5.7$ & $35.3 \pm 7.1$ & $55.1 \pm 8.6$ & $9.1 \pm 4.4$ & $49.9 \pm 8.2$ & $13.0 \pm 5.0$ & $-0.63_{-0.10}^{+0.09}$ & $-0.10_{-0.16}^{+0.08}$ & $\begin{array}{l}0.59_{-0.17}^{+0.18} \\
0\end{array}$ & $\mathrm{~N}$ & $\mathrm{~N}$ & $\ldots$ & 38.6 \\
\hline 6 & $\leqslant 2.0$ & $\ldots$ & $\ldots$ & $\ldots$ & $\ldots$ & $\ldots$ & $\ldots$ & & $\ldots$ & $\ldots$ & $\ldots$ & $\ldots$ & $\ldots$ & $\leqslant 37.0$ \\
\hline 7 & $\leqslant 0.6$ & $\ldots$ & $\ldots$ & $\ldots$ & & $\ldots$ & $\ldots$ & & & $\ldots$ & $\ldots$ & $\ldots$ & $\ldots$ & $\leqslant 36.5$ \\
\hline 8 & $12.6 \pm 5.0$ & $2.2 \pm 2.9$ & $6.3 \pm 3.8$ & $8.6 \pm 4.3$ & $4.0 \pm 3.4$ & $5.3 \pm 3.6$ & $6.8 \pm 4.0$ & $0.06_{-0.30}^{+0.30}$ & $-0.29_{-0.46}^{+0.34}$ & $0.19_{-0.30}^{+0.32}$ & $\ldots$ & $\ldots$ & $\ldots$ & 37.8 \\
\hline 9 & $\leqslant 1.5$ & $\ldots$ & $\ldots$ & $\ldots$ & $\ldots$ & $\ldots$ & $\ldots$ & $\ldots$ & $\ldots$ & $\ldots$ & $\ldots$ & $\ldots$ & $\ldots$ & $\leqslant 36.9$ \\
\hline 10 & $\leqslant 1.3$ & $\ldots$ & $\ldots$ & $\ldots$ & $\ldots$ & $\ldots$ & $\ldots$ & $\ldots$ & $\ldots$ & $\ldots$ & $\ldots$ & $\ldots$ & $\ldots$ & $\leqslant 36.8$ \\
\hline
\end{tabular}

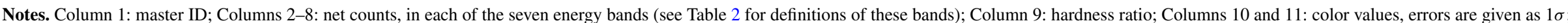

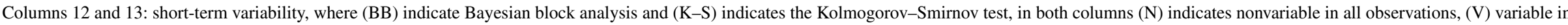

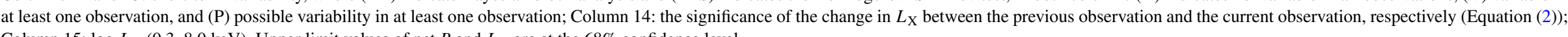
Column 15: $\log L_{\mathrm{X}}(0.3-8.0 \mathrm{keV})$. Upper limit values of net $B$ and $L_{\mathrm{X}}$ are at the $68 \%$ confidence level.

(This table is available in its entirety in a machine-readable form in the online journal. A portion is shown here for guidance regarding its form and content.) 
Table 6

Source Counts, Hardness Ratios, Color-Color Values, and Variability: Observation 2

\begin{tabular}{|c|c|c|c|c|c|c|c|c|c|c|c|c|c|c|}
\hline \multirow{2}{*}{$\begin{array}{l}\text { Master ID } \\
\text { (1) }\end{array}$} & \multicolumn{7}{|c|}{ Net Counts } & \multirow{2}{*}{$\begin{array}{l}\text { HR } \\
\text { (9) }\end{array}$} & \multirow{2}{*}{$\begin{array}{l}\text { C21 } \\
\text { (10) }\end{array}$} & \multirow{2}{*}{$\begin{array}{l}\text { C32 } \\
\text { (11) }\end{array}$} & \multicolumn{3}{|c|}{ Variability } & \multirow{2}{*}{$\begin{array}{c}\log L_{X} \\
(0.3-8.0 \mathrm{keV}) \\
(15)\end{array}$} \\
\hline & $\begin{array}{l}\text { B-Band } \\
\text { (2) }\end{array}$ & $\begin{array}{l}\text { S1-Band } \\
\text { (3) }\end{array}$ & $\begin{array}{l}\text { S2-Band } \\
\text { (4) }\end{array}$ & $\begin{array}{c}\text { S-Band } \\
(5)\end{array}$ & $\begin{array}{l}\text { H-Band } \\
\text { (6) }\end{array}$ & $\begin{array}{l}\text { Sc-Band } \\
\text { (7) }\end{array}$ & $\begin{array}{l}\text { Hc-Band } \\
\text { (8) }\end{array}$ & & & & $\begin{array}{l}\text { BB } \\
\text { (12) }\end{array}$ & $\begin{array}{l}\text { K-S } \\
\text { (13) }\end{array}$ & $\begin{array}{c}\text { Significance } \\
\text { (14) }\end{array}$ & \\
\hline 1 & $30.6 \pm 7.9$ & $1.3 \pm 3.4$ & $9.1 \pm 4.6$ & $10.4 \pm 5.2$ & $20.2 \pm 6.5$ & $9.0 \pm 4.6$ & $21.2 \pm 6.6$ & $0.35_{-0.20}^{+0.21}$ & $-0.48_{-0.83}^{+0.42}$ & $-0.30_{-0.22}^{+0.19}$ & $\mathrm{~N}$ & $\mathrm{~N}$ & 0.2 & 37.8 \\
\hline 2 & $64.6 \pm 10.8$ & $4.3 \pm 4.0$ & $45.5 \pm 8.1$ & $49.8 \pm 8.6$ & $14.9 \pm 6.0$ & $42.9 \pm 7.9$ & $18.0 \pm 6.4$ & $-0.47_{-0.12}^{+0.15}$ & $-0.88_{-0.37}^{+0.28}$ & $0.49_{-0.14}^{+0.19}$ & $\mathrm{~N}$ & $\mathrm{P}$ & 1.7 & 38.1 \\
\hline 3 & $\leqslant 6.6$ & $\ldots$ & $\ldots$ & $\ldots$ & $\ldots$ & $\ldots$ & $\ldots$ & $\ldots$ & $\ldots$ & $\ldots$ & $\ldots$ & $\ldots$ & 0.0 & $\leqslant 37.1$ \\
\hline 4 & $71.0 \pm 11.2$ & $23.3 \pm 6.2$ & $35.4 \pm 7.3$ & $58.7 \pm 9.1$ & $12.1 \pm 5.7$ & $52.3 \pm 8.5$ & $14.2 \pm 6.0$ & $-0.62_{-0.11}^{+0.13}$ & $-0.06_{-0.15}^{+0.09}$ & $0.43_{-0.13}^{+0.25}$ & $\mathrm{~N}$ & $\mathrm{P}$ & 1.3 & 38.1 \\
\hline 5 & $170.3 \pm 14.7$ & $48.7 \pm 8.3$ & $93.7 \pm 10.9$ & $142.3 \pm 13.2$ & $28.0 \pm 7.1$ & $120.1 \pm 12.2$ & $41.2 \pm 8.1$ & $-0.54_{-000}^{+0.07}$ & $-0.17_{-0.10}^{+0.06}$ & $0.55_{-011}^{+0.10}$ & $\mathrm{~N}$ & $\mathrm{~N}$ & 0.4 & 38.5 \\
\hline 6 & $\leqslant 1.8$ & $\ldots$ & $\ldots$ & $\ldots$ & $\ldots$ & $\ldots$ & $\ldots$ & $\ldots$ & $\ldots$ & $\ldots$ & $\ldots$ & $\ldots$ & 0.0 & $\leqslant 36.5$ \\
\hline 7 & $\leqslant 4.4$ & $\ldots$ & $\ldots$ & $\ldots$ & $\ldots$ & $\ldots$ & $\ldots$ & $\ldots$ & $\ldots$ & $\ldots$ & $\ldots$ & $\ldots$ & 0.0 & $\leqslant 36.9$ \\
\hline 8 & $32.2 \pm 7.7$ & $11.0 \pm 4.7$ & $23.0 \pm 6.2$ & $34.0 \pm 7.3$ & $-1.8 \pm 3.4$ & $28.9 \pm 6.7$ & $0.4 \pm 4.0$ & $-0.97_{-0.03}^{+0.06}$ & $-0.22_{-0.18}^{+0.16}$ & $1.30_{-046}^{+0.92}$ & $\mathrm{~N}$ & $\mathrm{~N}$ & 0.2 & 37.8 \\
\hline 9 & $\leqslant 8.6$ & $\ldots$ & $\ldots$ & $\ldots$ & $\ldots$ & $\ldots$ & $\ldots$ & $\ldots$ & $\ldots$ & $\ldots$ & $\ldots$ & $\ldots$ & 0.0 & $\leqslant 37.2$ \\
\hline 10 & $\leqslant 2.2$ & $\ldots$ & $\ldots$ & $\ldots$ & $\ldots$ & $\ldots$ & $\ldots$ & $\ldots$ & $\ldots$ & $\ldots$ & $\ldots$ & $\ldots$ & 0.0 & $\leqslant 36.6$ \\
\hline
\end{tabular}

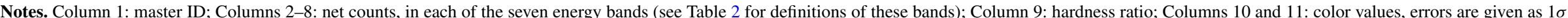

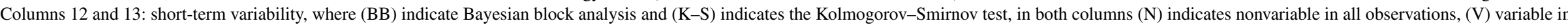

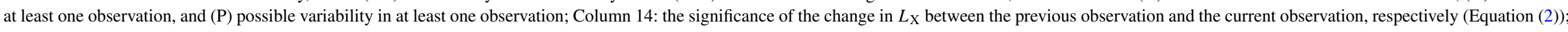
Column 15: $\log L_{\mathrm{X}}(0.3-8.0 \mathrm{keV})$. Upper limit values of net $B$ and $L_{\mathrm{X}}$ are at the $68 \%$ confidence level.

(This table is available in its entirety in a machine-readable form in the online journal. A portion is shown here for guidance regarding its form and content.)

Table 7

Source Counts, Hardness Ratios, Color-Color Values, and Variability: Observation 3

\begin{tabular}{|c|c|c|c|c|c|c|c|c|c|c|c|c|c|c|}
\hline \multirow{2}{*}{$\begin{array}{l}\text { Master ID } \\
\text { (1) }\end{array}$} & \multicolumn{7}{|c|}{ Net Counts } & \multirow{2}{*}{$\begin{array}{l}\text { HR } \\
\text { (9) }\end{array}$} & \multirow{2}{*}{$\begin{array}{l}\mathrm{C} 21 \\
(10)\end{array}$} & \multirow{2}{*}{$\begin{array}{l}\mathrm{C} 32 \\
\text { (11) }\end{array}$} & \multicolumn{3}{|c|}{ Variability } & \multirow{2}{*}{$\begin{array}{c}\log L_{X} \\
(0.3-8.0 \mathrm{keV}) \\
(15)\end{array}$} \\
\hline & $\begin{array}{l}\text { B-Band } \\
\text { (2) }\end{array}$ & $\begin{array}{l}\text { S1-Band } \\
\text { (3) }\end{array}$ & $\begin{array}{c}\text { S2-Band } \\
\text { (4) }\end{array}$ & $\begin{array}{c}\text { S-Band } \\
\text { (5) }\end{array}$ & $\begin{array}{c}\text { H-Band } \\
(6)\end{array}$ & $\begin{array}{c}\text { Sc-Band } \\
(7)\end{array}$ & $\begin{array}{c}\text { Hc-Band } \\
(8)\end{array}$ & & & & $\begin{array}{l}\text { BB } \\
\text { (12) }\end{array}$ & $\begin{array}{l}\text { K-S } \\
\text { (13) }\end{array}$ & $\begin{array}{c}\text { Significance } \\
(14)\end{array}$ & \\
\hline 1 & $22.5 \pm 6.2$ & $0.1 \pm 2.3$ & $11.1 \pm 4.6$ & $11.2 \pm 4.7$ & $11.3 \pm 4.7$ & $8.3 \pm 4.1$ & $14.8 \pm 5.2$ & $0.14_{-0.22}^{+0.22}$ & $-0.84_{-0.92}^{+0.46}$ & $0.06_{-0.19}^{+0.18}$ & $\mathrm{~N}$ & $\mathrm{~N}$ & 1.4 & 38.0 \\
\hline 2 & $37.1 \pm 7.5$ & $3.3 \pm 3.2$ & $20.1 \pm 5.7$ & $23.4 \pm 6.1$ & $13.7 \pm 5.1$ & $19.9 \pm 5.7$ & $16.4 \pm 5.4$ & $-0.24_{-0.17}^{+0.16}$ & $-0.55_{-0.29}^{+0.29}$ & $0.21_{-0.12}^{+0.20}$ & $\mathrm{~N}$ & $\mathrm{~N}$ & 1.4 & 38.3 \\
\hline 3 & $\leqslant 1.4$ & $\ldots$ & $\ldots$ & $\ldots$ & 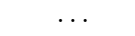 & $\ldots$ & $\ldots$ & $\ldots$ & $\ldots$ & & $\ldots$ & $\ldots$ & 0.0 & $\leqslant 36.8$ \\
\hline 4 & $36.6 \pm 7.5$ & $10.5 \pm 4.4$ & $21.9 \pm 5.9$ & $32.4 \pm 6.9$ & $4.2 \pm 3.8$ & $32.1 \pm 6.8$ & $3.7 \pm 3.8$ & $-0.87_{-0.09}^{+0.07}$ & $-0.11_{-0.17}^{+0.15}$ & $0.72_{-0.26}^{+0.35}$ & $\mathrm{~N}$ & $\mathrm{~N}$ & 1.0 & 38.2 \\
\hline 5 & $64.5 \pm 9.3$ & $17.5 \pm 5.3$ & $34.9 \pm 7.1$ & $52.4 \pm 8.4$ & $12.1 \pm 4.8$ & $47.9 \pm 8.1$ & $12.7 \pm 5.0$ & $-0.66_{-0.10}^{+0.09}$ & $-0.09_{-0.13}^{+0.13}$ & $0.51_{-0.15}^{+0.16}$ & $\mathrm{~N}$ & $\mathrm{~N}$ & 0.6 & 38.5 \\
\hline 6 & $\leqslant 2.1$ & $\ldots$ & $\ldots$ & $\ldots$ & $\ldots$ & $\ldots$ & $\ldots$ & $\ldots$ & $\ldots$ & $\ldots$ & $\cdots$ & $\cdots$ & 0.0 & $\leqslant 37.0$ \\
\hline 7 & $\leqslant 1.3$ & $\ldots$ & $\ldots$ & $\ldots$ & $\ldots$ & $\ldots$ & $\ldots$ & $\ldots$ & $\ldots$ & $\ldots$ & $\cdots$ & $\cdots$ & 0.0 & $\leqslant 36.8$ \\
\hline 8 & $12.4 \pm 5.0$ & $5.5 \pm 3.6$ & $4.3 \pm 3.4$ & $9.8 \pm 4.4$ & $2.6 \pm 3.2$ & $8.0 \pm 4.1$ & $3.5 \pm 3.4$ & $-0.49_{-0.30}^{+0.27}$ & $0.21_{-0.26}^{+0.33}$ & $0.21_{-0.42}^{+0.49}$ & $\cdots$ & $\ldots$ & 0.2 & 37.7 \\
\hline 9 & $\leqslant 5.7$ & $\ldots$ & $\ldots$ & $\ldots$ & $\ldots$ & $\ldots$ & $\ldots$ & $\ldots$ & $\ldots$ & $\ldots$ & $\ldots$ & $\ldots$ & 0.0 & $\leqslant 37.4$ \\
\hline 10 & $\leqslant 0.6$ & $\ldots$ & $\ldots$ & $\ldots$ & $\ldots$ & $\ldots$ & $\ldots$ & $\cdots$ & $\ldots$ & $\ldots$ & $\ldots$ & $\ldots$ & 0.0 & $\leqslant 36.4$ \\
\hline
\end{tabular}

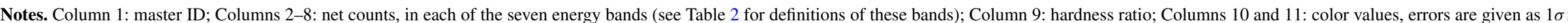

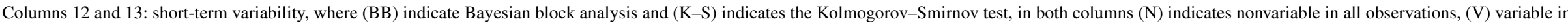

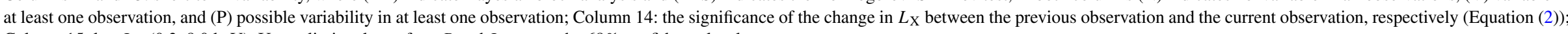
Column 15: $\log L_{\mathrm{X}}(0.3-8.0 \mathrm{keV})$. Upper limit values of net $B$ and $L_{\mathrm{X}}$ are at the $68 \%$ confidence level.

(This table is available in its entirety in a machine-readable form in the online journal. A portion is shown here for guidance regarding its form and content.) 
Table 8

Source Counts, Hardness Ratios, Color-Color Values, and Variability: Observation 4

\begin{tabular}{|c|c|c|c|c|c|c|c|c|c|c|c|c|c|c|}
\hline \multirow{2}{*}{$\begin{array}{l}\text { Master ID } \\
\text { (1) }\end{array}$} & \multirow[b]{2}{*}{$\begin{array}{c}\text { B-Band } \\
\text { (2) }\end{array}$} & \multirow[b]{2}{*}{$\begin{array}{c}\text { S1-Band } \\
\text { (3) }\end{array}$} & \multirow[b]{2}{*}{$\begin{array}{c}\text { S2-Band } \\
(4)\end{array}$} & \multirow{2}{*}{$\begin{array}{c}\text { Net Counts } \\
\text { S-Band } \\
(5)\end{array}$} & \multirow[b]{2}{*}{$\begin{array}{c}\text { H-Band } \\
(6)\end{array}$} & \multirow[b]{2}{*}{$\begin{array}{c}\text { Sc-Band } \\
(7)\end{array}$} & \multirow[b]{2}{*}{$\begin{array}{c}\text { Hc-Band } \\
(8)\end{array}$} & \multirow{2}{*}{$\begin{array}{l}\text { HR } \\
(9) \\
\end{array}$} & \multirow{2}{*}{$\begin{array}{l}\text { C21 } \\
(10) \\
\end{array}$} & \multirow{2}{*}{$\begin{array}{l}\text { C32 } \\
(11) \\
\end{array}$} & \multicolumn{3}{|c|}{ Variability } & \multirow{2}{*}{$\begin{array}{c}\log L_{X} \\
(0.3-8.0 \mathrm{keV}) \\
(15) \\
\end{array}$} \\
\hline & & & & & & & & & & & $\begin{array}{l}\text { BB } \\
(12)\end{array}$ & $\begin{array}{l}\mathrm{K}-\mathrm{S} \\
(13) \\
\end{array}$ & $\begin{array}{c}\text { Significance } \\
(14)\end{array}$ & \\
\hline 1 & $22.1 \pm 6.1$ & $-0.8 \pm 1.9$ & $8.4 \pm 4.1$ & $7.6 \pm 4.1$ & $14.5 \pm 5.1$ & $5.2 \pm 3.6$ & $17.4 \pm 5.4$ & $0.50_{-0.18}^{+0.21}$ & $-1.15_{-0.99}^{+0.54}$ & $-0.21_{-0.19}^{+0.18}$ & $\mathrm{~N}$ & $\mathrm{~N}$ & 0.0 & 38.0 \\
\hline 2 & $97.9 \pm 11.3$ & $11.4 \pm 4.7$ & $68.0 \pm 9.4$ & $79.4 \pm 10.2$ & $18.4 \pm 5.8$ & $69.9 \pm 9.5$ & $27.8 \pm 6.7$ & $-0.50_{-0.09}^{+0.08}$ & $-0.62_{-0.16}^{+0.13}$ & $0.61_{-0.13}^{+0.11}$ & $\mathrm{~N}$ & $\mathrm{~N}$ & 0.5 & 38.3 \\
\hline 3 & $\leqslant 0.9$ & $\ldots$ & $\ldots$ & $\ldots$ & $\ldots$ & $\ldots$ & $\ldots$ & $\ldots$ & $\ldots$ & $\ldots$ & $\ldots$ & $\cdots$ & 0.0 & $\leqslant 36.3$ \\
\hline 4 & $45.5 \pm 8.4$ & $11.4 \pm 4.7$ & $24.8 \pm 6.3$ & $36.1 \pm 7.4$ & $9.4 \pm 4.7$ & $29.8 \pm 6.7$ & $13.8 \pm 5.3$ & $-0.44_{-0.16}^{+0.13}$ & $-0.20_{-0.17}^{+0.16}$ & $0.43_{-0.16}^{+0.21}$ & $\mathrm{~N}$ & $\mathrm{~N}$ & 2.0 & 38.0 \\
\hline 5 & $131.6 \pm 12.8$ & $42.5 \pm 7.7$ & $63.8 \pm 9.2$ & $106.3 \pm 11.5$ & $25.4 \pm 6.5$ & $88.4 \pm 10.6$ & $31.0 \pm 7.0$ & $-0.53_{-0.08}^{+0.07}$ & $-0.06_{-0.10}^{+0.08}$ & $0.42_{-0.10}^{+0.11}$ & $\mathrm{~N}$ & $\mathrm{Y}$ & 0.5 & 38.4 \\
\hline 6 & $\leqslant 10.4$ & $\ldots$ & $\ldots$ & $\ldots$ & $\ldots$ & $\ldots$ & $\ldots$ & $\ldots$ & $\ldots$ & $\ldots$ & $\cdots$ & $\cdots$ & 0.8 & $\leqslant 37.3$ \\
\hline 7 & $\leqslant 2.6$ & $\ldots$ & $\ldots$ & $\ldots$ & $\ldots$ & $\ldots$ & $\ldots$ & $\ldots$ & $\ldots$ & $\ldots$ & $\ldots$ & $\ldots$ & 0.0 & $\leqslant 36.7$ \\
\hline 8 & $23.8 \pm 6.7$ & $3.4 \pm 3.4$ & $17.3 \pm 5.4$ & $20.7 \pm 6.0$ & $3.1 \pm 3.8$ & $17.1 \pm 5.4$ & $5.7 \pm 4.3$ & $-0.59_{-0.22}^{+0.21}$ & $-0.54_{-0.37}^{+0.30}$ & $0.67_{-0.32}^{+0.56}$ & $\mathrm{~N}$ & $\mathrm{~N}$ & 0.3 & 37.7 \\
\hline 9 & $\leqslant 5.5$ & $\ldots$ & $\ldots$ & ( & $\ldots$ & $\ldots$ & $\ldots$ & $\ldots$ & $\ldots$ & $\ldots$ & $\ldots$ & $\ldots$ & 0.0 & $\leqslant 37.1$ \\
\hline 10 & $\leqslant 2.3$ & $\cdots$ & $\ldots$ & & $\ldots$ & $\ldots$ & $\ldots$ & $\ldots$ & $\ldots$ & $\ldots$ & & $\ldots$ & 0.0 & $\leqslant 36.7$ \\
\hline
\end{tabular}

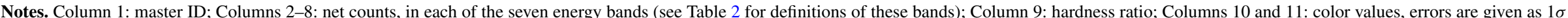

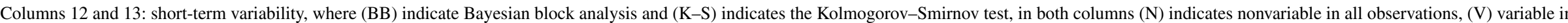

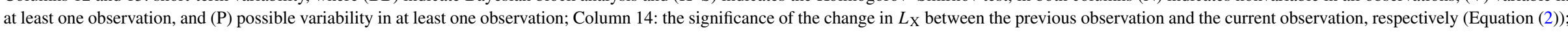
Column 15: $\log L_{\mathrm{X}}(0.3-8.0 \mathrm{keV})$. Upper limit values of net $B$ and $L_{\mathrm{X}}$ are at the $68 \%$ confidence level.

(This table is available in its entirety in a machine-readable form in the online journal. A portion is shown here for guidance regarding its form and content.)

Table 9

Source Counts, Hardness Ratios, Color-Color Values, and Variability: Observation 5

\begin{tabular}{|c|c|c|c|c|c|c|c|c|c|c|c|c|c|c|}
\hline \multirow{2}{*}{$\begin{array}{l}\text { Master ID } \\
\text { (1) }\end{array}$} & \multirow[b]{2}{*}{$\begin{array}{l}\text { B-Band } \\
\text { (2) }\end{array}$} & \multirow[b]{2}{*}{$\begin{array}{l}\text { S1-Band } \\
\text { (3) }\end{array}$} & \multirow[b]{2}{*}{$\begin{array}{l}\text { S2-Band } \\
\text { (4) }\end{array}$} & \multirow{2}{*}{$\begin{array}{c}\text { Net Counts } \\
\text { S-Band } \\
\text { (5) }\end{array}$} & \multirow[b]{2}{*}{$\begin{array}{l}\text { H-Band } \\
\text { (6) }\end{array}$} & \multirow[b]{2}{*}{$\begin{array}{l}\text { Sc-Band } \\
\text { (7) }\end{array}$} & \multirow[b]{2}{*}{$\begin{array}{l}\text { Hc-Band } \\
\text { (8) }\end{array}$} & \multirow{2}{*}{$\begin{array}{l}\text { HR } \\
\text { (9) }\end{array}$} & \multirow{2}{*}{$\begin{array}{l}\text { C21 } \\
(10) \\
\end{array}$} & \multirow{2}{*}{$\begin{array}{l}\text { C32 } \\
(11) \\
\end{array}$} & \multicolumn{3}{|c|}{ Variability } & \multirow{2}{*}{$\begin{array}{c}\log L_{X} \\
(0.3-8.0 \mathrm{keV}) \\
(15)\end{array}$} \\
\hline & & & & & & & & & & & $\begin{array}{l}\text { BB } \\
\text { (12) }\end{array}$ & $\begin{array}{l}\text { K-S } \\
\text { (13) }\end{array}$ & $\begin{array}{c}\text { Significance } \\
\text { (14) }\end{array}$ & \\
\hline 1 & $49.4 \pm 9.0$ & $0.4 \pm 3.2$ & $13.1 \pm 5.1$ & $13.6 \pm 5.6$ & $35.8 \pm 7.5$ & $10.6 \pm 4.8$ & $37.1 \pm 7.7$ & $0.51_{-0.14}^{+0.15}$ & $-0.76_{-0.85}^{+0.47}$ & $-0.38_{-0.17}^{+0.14}$ & $\mathrm{~N}$ & $\mathrm{~N}$ & 0.4 & 38.0 \\
\hline 2 & $129.3 \pm 13.2$ & $22.1 \pm 6.3$ & $79.4 \pm 10.2$ & $101.5 \pm 11.5$ & $27.8 \pm 7.1$ & $83.1 \pm 10.4$ & $44.1 \pm 8.4$ & $-0.36_{-0.09}^{+0.08}$ & $-0.45_{-0.12}^{+0.11}$ & $0.48_{-0.11}^{+0.11}$ & $\mathrm{~N}$ & $\mathrm{~N}$ & 1.3 & 38.4 \\
\hline 3 & $\leqslant 2.3$ & $\ldots$ & $\ldots$ & $\ldots$ & $\ldots$ & $\ldots$ & $\ldots$ & $\ldots$ & $\ldots$ & $\ldots$ & $\ldots$ & $\ldots$ & 0.0 & $\leqslant 36.6$ \\
\hline 4 & $76.2 \pm 10.6$ & $22.0 \pm 6.2$ & $41.7 \pm 7.8$ & $63.7 \pm 9.5$ & $12.6 \pm 5.6$ & $53.7 \pm 8.7$ & $18.9 \pm 6.3$ & $-0.53_{-0.12}^{+0.11}$ & $-0.19_{-0.10}^{+0.14}$ & $0.53_{-0.16}^{+0.19}$ & $\mathrm{~N}$ & $\mathrm{~N}$ & 1.9 & 38.2 \\
\hline 5 & $204.7 \pm 15.8$ & $49.8 \pm 8.4$ & $118.2 \pm 12.0$ & $168.0 \pm 14.3$ & $36.7 \pm 7.6$ & $140.7 \pm 13.1$ & $54.1 \pm 8.9$ & $-0.49_{-0.07}^{+0.06}$ & $-0.28_{-0.07}^{+0.08}$ & $\begin{array}{r}0.53_{-0.09}^{+0.09} \\
0\end{array}$ & $\mathrm{~N}$ & $\mathrm{~N}$ & 2.7 & 38.6 \\
\hline 6 & $\leqslant 1.8$ & $\ldots$ & $\ldots$ & $\ldots$ & $\ldots$ & $\ldots$ & $\ldots$ & $\ldots$ & $\ldots$ & $\ldots$ & $\ldots$ & $\ldots$ & 1.2 & $\leqslant 36.5$ \\
\hline 7 & $\leqslant 2.1$ & $\ldots$ & $\ldots$ & $\ldots$ & $\ldots$ & $\ldots$ & $\ldots$ & $\ldots$ & $\ldots$ & $\ldots$ & $\ldots$ & $\ldots$ & 0.0 & $\leqslant 36.6$ \\
\hline 8 & $26.4 \pm 7.4$ & $6.0 \pm 4.1$ & $16.9 \pm 5.6$ & $22.9 \pm 6.5$ & $3.5 \pm 4.4$ & $23.5 \pm 6.3$ & $2.8 \pm 4.4$ & $-0.88_{-012}^{+0.11}$ & $-0.32_{-0.30}^{+0.21}$ & $0.59_{-035}^{+0.59}$ & $\mathrm{~N}$ & $\mathrm{~N}$ & 0.1 & 37.7 \\
\hline 9 & $21.2 \pm 7.1$ & $6.3 \pm 4.3$ & $12.5 \pm 5.1$ & $18.8 \pm 6.2$ & $2.4 \pm 4.3$ & $19.3 \pm 5.9$ & $2.5 \pm 4.4$ & $-0.87_{-0.13}^{+0.12}$ & $-0.19_{-0.29}^{+0.24}$ & $0.56_{-0.40}^{+0.70}$ & $\mathrm{~N}$ & $\mathrm{~N}$ & 2.3 & 37.6 \\
\hline 10 & $\leqslant 7.3$ & $\ldots$ & $\ldots$ & $\ldots$ & $\ldots$ & $\ldots$ & $\ldots$ & $\ldots^{-0.13}$ & $\ldots$ & $\ldots$ & $\ldots$ & $\ldots$ & 0.0 & $\leqslant 37.1$ \\
\hline
\end{tabular}

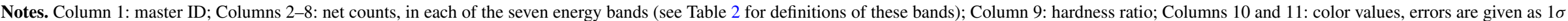

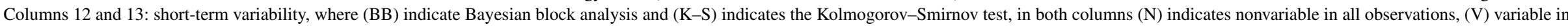

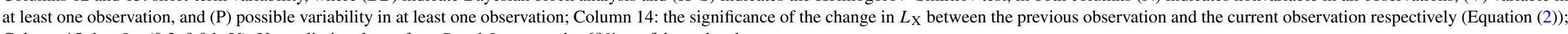
Column 15: $\log L_{\mathrm{X}}(0.3-8.0 \mathrm{keV})$. Upper limit values of net $B$ and $L_{\mathrm{X}}$ are at the $68 \%$ confidence level.

(This table is available in its entirety in a machine-readable form in the online journal. A portion is shown here for guidance regarding its form and content.) 
also indicated by a horizontal dashed green line. (Online-only: In instances where the source was not detected in the co-added observation, a blue line indicates the upper limit of the source luminosity.)

Figure 8 presents the $L_{X}-\mathrm{HR}$ plots for sources with measured hardness ratios in at least two observations. Each point shows the X-ray luminosity and hardness ratio value of a source during each pointing, as well as the values derived from the co-added observation. Each point is labeled (and color coded in the online-only version as magenta, green, blue, red, cyan, and dark green) to indicate observations $1-6$, respectively, and the black solid box represents the co-added observation value. Similarly, Figure 9 presents the color-color values for sources with measured color-color values in at least two observations, where again individual observations are labeled and color coded (following the same color scheme as in Figure 8 in the onlineonly version), with the co-added observation indicated by the in black solid box.

\section{DISCUSSION}

\subsection{X-Ray Source Population}

In the previous sections the data analysis methods, used to determine the properties of the X-ray binary population of NGC 4278, have been presented. From the six individual Chandra pointings, taken between 2005 February and 2007 February, a co-added observation, totaling an exposure time of $458 \mathrm{ks}$, has been produced. From this deep observation of the galaxy, $236 \mathrm{X}$ ray point sources have been detected in the region overlapped by all of the individual pointings, with 180 of these sources residing within the $D_{25}$ ellipse of the system. These 236 sources are presented in Figure 3 where a raw, full band image from the co-added observation, with the overlap region and $D_{25}$ ellipse overlaid, is presented in the main image, with source regions also indicated. The two following images present the central region of the galaxy, where the dense population of sources can be more clearly seen. Of these 236 sources, based on the hardband ChaMP+CDF $\log N-\log S$ relation (Kim et al. 2007b), $\sim 29$ sources detected in the co-added observation are expected to be objects not associated with NGC 4278. Within the $D_{25}$ ellipse of the galaxy, it is expected that $\sim 12$ of these sources are background objects. In Figure 4, the number of expected background objects is indicated in the X-ray source number density profile of the galaxy and appears consistent with the flattening of the profile at larger radii.

The total number of LMXBs residing in NGC 4278 is much greater than that of NGC 3379 (notwithstanding the $90 \%$ completeness limit of $L_{X} \sim 5 \times 10^{36} \mathrm{erg} \mathrm{s}^{-1}$ compared to $L_{X} \sim 1 \times 10^{37} \mathrm{erg} \mathrm{s}^{-1}$ in NGC 4278), where only 98 sources were detected within the $D_{25}$ ellipse of the galaxy, compared to 180 in the $D_{25}$ ellipse of NGC 4278. This increase in the total LMXB population in NGC 4278 follows the known correlation of increasing X-ray luminosity arising from LMXBs (normalized by $\left.L_{\mathrm{B}}\right)$ with increasing GC-specific frequency $\left(S_{\mathrm{GC}}\right.$; e.g., Kim et al. 2006; Irwin 2005). Such a relationship confirms the importance of LMXB formation in GCs, and through the deep observations that have presented here and in Brassington et al. (2008) this correlation will be further investigated in a forthcoming paper, where the hypothesis that all LMXBs were formed in GCs will be tested.

The X-ray luminosity of the sources detected within NGC 4278 ranges from $3.5 \times 10^{36} \mathrm{erg} \mathrm{s}^{-1}$ (with $3 \sigma$ upper limit $\leqslant 1 \times 10^{37} \mathrm{erg} \mathrm{s}^{-1}$ ) up to $\sim 2 \times 10^{40} \mathrm{erg} \mathrm{s}^{-1}$, where the brightest source, 117 , lies at the nucleus of the galaxy and has been classed as a LINER with $\mathrm{H} \alpha$ emission (Ho et al. 1997). The full X-ray data analysis of this source from these six observations will be reported in a forthcoming paper. Apart from this central source, no LMXBs exceeding $1 \times 10^{39} \mathrm{erg} \mathrm{s}^{-1}$ have been detected in this galaxy, unlike NGC 3379, where a ULX source with a co-added X-ray luminosity of $\sim 2 \times 10^{39} \mathrm{erg} \mathrm{s}^{-1}$ was discovered. This lack of ULXs in NGC 4278 is unsurprising as it is rare to find such a high-luminosity object (external to the nuclear region) in E and S0 galaxies (Irwin et al. 2004).

The $L_{\mathrm{X}}$ distribution of all of the detected X-ray sources within NGC 4278 is shown in Figure 10, where the GC associations are also indicated. In this figure the main histogram presents the calculated $L_{\mathrm{X}}$ values from all sources (with $1 \sigma$ upper limits from the co-added observation provided for sources only detected in a single observation), except for the central bright source 117. The bottom left histogram presents these same sources, but for those with $\mathrm{S} / \mathrm{N}<3,3 \sigma$ upper limits are shown, these upper limit values are then presented separately in the bottom right histogram. From the main figure it can be seen that the majority of sources detected from this observation lie in the luminosity range of $1 \times 10^{37} \mathrm{erg} \mathrm{s}^{-1}$ to $6 \times 10^{37} \mathrm{erg} \mathrm{s}^{-1}$, with a mode luminosity of $\sim 1.5 \times 10^{37} \mathrm{erg} \mathrm{s}^{-1}$ and with source incompleteness beginning to affect the source distribution $L_{\mathrm{X}} \leqslant$ $1 \times 10^{37} \mathrm{erg} \mathrm{s}^{-1}$. From the histogram including $3 \sigma$ upper limit values, this mode value remains the same, as does source incompleteness.

In the forthcoming paper Kim et al. (2009), the XLF of NGC 4278 has been investigated, and a correction to allow for source incompleteness has been applied. Some preliminary results, investigating the XLF of NGC 4278, alongside NGC 3379, have been reported in Kim et al. (2006), where sources, down to a $90 \%$ completeness limit of $L_{X} \sim 3 \times 10^{37} \mathrm{erg} \mathrm{s}^{-1}$, from the first two observations, have been detected. From the even greater sensitivity afforded to us by combining the six separate pointings, we can investigate the XLF close to the X-ray luminosity range of normal Galactic LMXBs. Previously, this has only been possible for the nearby radio galaxy Centaurus A (NGC 5128), where the XLF has been measured down to $\sim 2 \times 10^{36} \mathrm{erg} \mathrm{s}^{-1}$ (Kraft et al. 2001; Voss \& Gilfanov 2006). With our greater sensitivity we can compare our results to these studies, allowing us to investigate the shape of the lowluminosity LMXB XLF, although it should be noted that both NGC 3379 and NGC 4278 are much more "normal" galaxies than Centaurus A.

In addition to the X-ray point sources that have been presented in this catalog, the optical sources within NGC 4278 have also been identified. These were detected in a WFPC2 HST observation, where 266 confirmed GCs have been identified. From these 266 sources, 39 GC-LMXB, with separations $<0$." 6 , have been detected. From Figure 10, it can be seen that these 39 GC-LMXB sources appear to predominantly lie at the high X-ray luminosity end of this distribution. The luminosity function of these GC-LMXB sources from both galaxies and its implications for the understanding of LMXB evolution is discussed in Kim et al. (2009), where the XLFs of both GC and field LMXBs has been compared.

\subsection{X-Ray Colors}

In Figure 11 the LMXB population color-color diagram, based on the photometry of the co-added observation, is presented. In the top panel color-color values are plotted, with the sources divided into luminosity bins, with symbols of each bin 
Table 10

Source Counts, Hardness Ratios, Color-Color Values, and Variability: Observation 6

\begin{tabular}{|c|c|c|c|c|c|c|c|c|c|c|c|c|c|c|}
\hline Master ID & & & & Net Counts & & & & HR & $\mathrm{C} 21$ & C32 & & Varia & lity & $\log L_{X}$ \\
\hline (1) & $\begin{array}{l}\text { B-Band } \\
\text { (2) }\end{array}$ & $\begin{array}{l}\text { S1-Band } \\
\text { (3) }\end{array}$ & $\begin{array}{l}\text { S2-Band } \\
\text { (4) }\end{array}$ & $\begin{array}{l}\text { S-Band } \\
\text { (5) }\end{array}$ & $\begin{array}{l}\text { H-Band } \\
(6)\end{array}$ & $\begin{array}{l}\text { Sc-Band } \\
(7)\end{array}$ & $\begin{array}{c}\text { Hc-Band } \\
(8)\end{array}$ & (9) & (10) & $(11)$ & $\begin{array}{l}\text { BB } \\
(12)\end{array}$ & $\begin{array}{l}\text { K-S } \\
\text { (13) }\end{array}$ & $\begin{array}{l}\text { Significance } \\
\text { (14) }\end{array}$ & $\begin{array}{c}(0.3-8.0 \mathrm{keV} \\
(15)\end{array}$ \\
\hline 1 & $10.1 \pm 5.4$ & $-1.0 \pm 2.3$ & $7.3 \pm 4.1$ & $6.4 \pm 4.3$ & $3.7 \pm 4.0$ & $5.9 \pm 4.0$ & $4.2 \pm 4.1$ & $-0.33_{-0.37}^{+0.41}$ & $-0.92_{-0.91}^{+0.54}$ & $0.27_{-0.38}^{+0.51}$ & & $\ldots$ & 2.1 & 37.6 \\
\hline 2 & $47.4 \pm 8.4$ & $9.8 \pm 4.4$ & $24.2 \pm 6.2$ & $34.0 \pm 7.1$ & $13.3 \pm 5.2$ & $31.0 \pm 6.8$ & $14.9 \pm 5.4$ & $-0.46_{-0.14}^{+0.37}$ & $-0.16_{-0.20}^{+0.915}$ & $\begin{array}{c}-0.38 \\
0.31_{-0.16}^{+0.16}\end{array}$ & $\mathrm{~N}$ & $\mathrm{~N}$ & 1.1 & 38.3 \\
\hline 3 & $\leqslant 3.0$ & $\ldots$ & $\ldots$ & & $\ldots$ & $\ldots$ & $\ldots$ & $\ldots$ & $\ldots$ & $\ldots$ & $\ldots$ & $\ldots$ & 0.0 & $\leqslant 37.1$ \\
\hline 4 & $20.7 \pm 6.3$ & $3.8 \pm 3.4$ & $13.5 \pm 5.0$ & $17.3 \pm 5.6$ & $3.4 \pm 3.8$ & $13.2 \pm 5.0$ & $4.9 \pm 4.1$ & $-0.60_{-023}^{+0.23}$ & $-0.32_{-0.32}^{+0.27}$ & $0.56_{-032}^{+0.49}$ & $\mathrm{~N}$ & $\mathrm{~N}$ & 1.8 & 37.9 \\
\hline 5 & $85.8 \pm 10.7$ & $22.6 \pm 6.0$ & $51.1 \pm 8.3$ & $73.7 \pm 9.8$ & $12.1 \pm 5.1$ & $58.5 \pm 8.9$ & $20.9 \pm 6.1$ & $-0.54_{-0.10}^{+0.09}$ & $-0.20_{-0.12}^{+0.10}$ & $0.66_{-0.17}^{+0.14}$ & $\mathrm{~N}$ & $\mathrm{~N}$ & 1.1 & 38.5 \\
\hline 6 & $\leqslant 4.7$ & $\ldots$ & $\ldots$ & $\ldots$ & $\ldots$ & $\ldots$ & $\ldots$ & $\ldots$ & $\ldots$ & $\ldots$ & $\ldots$ & $\ldots$ & 0.0 & $\leqslant 37.3$ \\
\hline 7 & $6.8 \pm 4.6$ & $2.1 \pm 2.9$ & $2.6 \pm 3.2$ & $4.7 \pm 3.8$ & $2.1 \pm 3.4$ & $1.6 \pm 2.9$ & $3.7 \pm 3.8$ & $0.34_{-0.28}^{+0.39}$ & $0.03_{-0.62}^{+0.59}$ & $0.08_{-0.67}^{+0.75}$ & $\ldots$ & $\ldots$ & 1.4 & 37.4 \\
\hline 8 & $21.7 \pm 6.2$ & $7.4 \pm 4.0$ & $8.9 \pm 4.3$ & $16.3 \pm 5.3$ & $5.5 \pm 4.0$ & $11.8 \pm 4.7$ & $9.2 \pm 4.6$ & $-0.23_{-0.24}^{+0.22}$ & $0.08_{-0.21}^{+0.21}$ & $0.24_{-0.27}^{+0.30}$ & $\mathrm{~N}$ & $\mathrm{~N}$ & 1.3 & 37.9 \\
\hline 9 & $\leqslant 7.8$ & $\ldots$ & $\ldots$ & $\ldots$ & $\ldots$ & $\ldots$ & $\ldots$ & $\ldots^{-0.24}$ & $\ldots$ & $\ldots$ & $\ldots$ & $\ldots$ & 1.1 & $\leqslant 37.5$ \\
\hline 10 & $\leqslant 4.2$ & $\cdots$ & $\cdots$ & $\ldots$ & $\cdots$ & $\ldots$ & $\ldots$ & $\cdots$ & $\ldots$ & $\cdots$ & $\cdots$ & $\ldots$ & 0.0 & $\leqslant 37.2$ \\
\hline
\end{tabular}

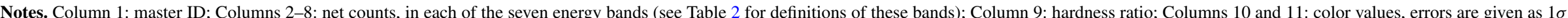

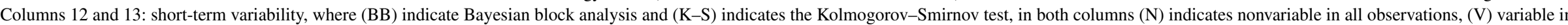

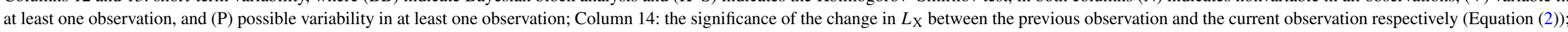
Column 15: $\log L_{\mathrm{X}}(0.3-8.0 \mathrm{keV})$. Upper limit values of net $B$ and $L_{\mathrm{X}}$ are at the $68 \%$ confidence level.

(This table is available in its entirety in a machine-readable form in the online journal. A portion is shown here for guidance regarding its form and content.) 
Table 11

Properties of Optical Sources that are Correlated with an X-Ray Point Source

\begin{tabular}{|c|c|c|c|c|c|}
\hline Master ID & (2) & (3) & $\begin{array}{l}V-I \\
(4)\end{array}$ & $\begin{array}{c}\text { GC Separation } \\
\text { (arcsec) } \\
(5)\end{array}$ & Ratio \\
\hline 51 & $21.21 \pm 0.01$ & $20.11 \pm 0.01$ & $1.11 \pm 0.02$ & 0.40 & 1.02 \\
\hline 63 & $22.31 \pm 0.02$ & $21.17 \pm 0.03$ & $1.14 \pm 0.03$ & 0.48 & 1.73 \\
\hline 68 & $20.25 \pm 0.01$ & $19.21 \pm 0.01$ & $1.04 \pm 0.01$ & 0.38 & 1.45 \\
\hline 76 & $22.75 \pm 0.03$ & $21.66 \pm 0.05$ & $1.09 \pm 0.06$ & 0.26 & 1.01 \\
\hline 81 & $22.10 \pm 0.02$ & $20.94 \pm 0.04$ & $1.16 \pm 0.04$ & 0.23 & 0.70 \\
\hline 83 & $21.63 \pm 0.01$ & $20.63 \pm 0.02$ & $1.00 \pm 0.02$ & 0.19 & 0.76 \\
\hline 84 & $22.14 \pm 0.02$ & $20.91 \pm 0.03$ & $1.23 \pm 0.04$ & 0.19 & 0.72 \\
\hline 85 & $22.29 \pm 0.02$ & $21.39 \pm 0.05$ & $0.90 \pm 0.05$ & 0.33 & 1.22 \\
\hline 96 & $20.96 \pm 0.01$ & $19.94 \pm 0.01$ & $1.03 \pm 0.02$ & 0.27 & 1.16 \\
\hline 98 & $22.68 \pm 0.04$ & $21.71 \pm 0.06$ & $0.97 \pm 0.07$ & 0.18 & 0.70 \\
\hline 108 & $21.64 \pm 0.01$ & $20.54 \pm 0.02$ & $1.10 \pm 0.02$ & 0.35 & 1.27 \\
\hline 118 & $21.97 \pm 0.02$ & $20.91 \pm 0.03$ & $1.06 \pm 0.04$ & 0.24 & 0.98 \\
\hline 134 & $23.80 \pm 0.11$ & $22.62 \pm 0.13$ & $1.18 \pm 0.17$ & 0.13 & 0.39 \\
\hline 148 & $22.89 \pm 0.04$ & $21.69 \pm 0.06$ & $1.19 \pm 0.07$ & 0.11 & 0.46 \\
\hline 153 & $22.66 \pm 0.03$ & $21.59 \pm 0.05$ & $1.08 \pm 0.06$ & 0.49 & 1.10 \\
\hline 154 & $20.61 \pm 0.01$ & $19.47 \pm 0.01$ & $1.14 \pm 0.01$ & 0.04 & 0.15 \\
\hline 161 & $23.34 \pm 0.04$ & $22.11 \pm 0.06$ & $1.24 \pm 0.07$ & 0.17 & 0.35 \\
\hline 162 & $23.31 \pm 0.06$ & $22.06 \pm 0.07$ & $1.26 \pm 0.09$ & 0.13 & 0.45 \\
\hline 163 & $21.80 \pm 0.02$ & $20.68 \pm 0.02$ & $1.12 \pm 0.03$ & 0.09 & 0.39 \\
\hline 165 & $22.53 \pm 0.02$ & $21.30 \pm 0.03$ & $1.23 \pm 0.04$ & 0.14 & 0.45 \\
\hline 167 & $22.06 \pm 0.02$ & $20.83 \pm 0.02$ & $1.23 \pm 0.03$ & 0.44 & 1.04 \\
\hline 172 & $21.40 \pm 0.01$ & $20.41 \pm 0.02$ & $0.98 \pm 0.02$ & 0.22 & 0.45 \\
\hline 173 & $23.17 \pm 0.04$ & $22.18 \pm 0.07$ & $0.99 \pm 0.08$ & 0.53 & 0.76 \\
\hline 174 & $21.40 \pm 0.01$ & $20.31 \pm 0.01$ & $1.09 \pm 0.02$ & 0.26 & 0.67 \\
\hline 179 & $21.73 \pm 0.01$ & $20.60 \pm 0.02$ & $1.13 \pm 0.02$ & 0.34 & 1.19 \\
\hline 185 & $22.30 \pm 0.02$ & $21.15 \pm 0.03$ & $1.15 \pm 0.03$ & 0.07 & 0.32 \\
\hline 187 & $24.19 \pm 0.08$ & $22.87 \pm 0.11$ & $1.32 \pm 0.14$ & 0.29 & 1.11 \\
\hline 190 & $22.42 \pm 0.02$ & $21.25 \pm 0.03$ & $1.16 \pm 0.04$ & 0.20 & 0.72 \\
\hline 191 & $22.83 \pm 0.03$ & $21.81 \pm 0.04$ & $1.02 \pm 0.05$ & 0.08 & 0.28 \\
\hline 193 & $24.07 \pm 0.07$ & $23.14 \pm 0.11$ & $0.93 \pm 0.13$ & 0.02 & 0.06 \\
\hline 194 & $21.75 \pm 0.01$ & $20.78 \pm 0.02$ & $0.97 \pm 0.03$ & 0.15 & 0.64 \\
\hline 199 & $22.14 \pm 0.02$ & $21.08 \pm 0.03$ & $1.06 \pm 0.03$ & 0.16 & 0.40 \\
\hline 202 & $23.08 \pm 0.03$ & $21.88 \pm 0.04$ & $1.20 \pm 0.05$ & 0.35 & 1.32 \\
\hline 203 & $22.30 \pm 0.02$ & $21.16 \pm 0.03$ & $1.14 \pm 0.03$ & 0.28 & 0.91 \\
\hline 208 & $22.65 \pm 0.02$ & $21.71 \pm 0.04$ & $0.94 \pm 0.04$ & 0.27 & 0.85 \\
\hline 214 & $21.34 \pm 0.01$ & $20.28 \pm 0.01$ & $1.06 \pm 0.02$ & 0.16 & 0.33 \\
\hline 215 & $22.79 \pm 0.02$ & $21.93 \pm 0.04$ & $0.86 \pm 0.05$ & 0.36 & 0.93 \\
\hline 216 & $21.32 \pm 0.01$ & $20.35 \pm 0.02$ & $0.97 \pm 0.02$ & 0.37 & 1.39 \\
\hline 217 & $21.03 \pm 0.01$ & $20.14 \pm 0.01$ & $0.89 \pm 0.01$ & 0.48 & 1.38 \\
\hline 117 & $19.39 \pm 0.02$ & $18.58 \pm 0.03$ & $0.81 \pm 0.03$ & 0.09 & 0.44 \\
\hline 128 & $24.15 \pm 0.22$ & $22.80 \pm 0.24$ & $1.35 \pm 0.33$ & 0.51 & 1.96 \\
\hline 129 & $24.43 \pm 0.28$ & $23.32 \pm 0.38$ & $1.12 \pm 0.47$ & 0.16 & 0.56 \\
\hline 147 & $24.00 \pm 0.14$ & $23.28 \pm 0.29$ & $0.73 \pm 0.32$ & 0.45 & 1.86 \\
\hline 189 & $19.73 \pm 0.01$ & $18.52 \pm 0.01$ & $1.21 \pm 0.01$ & 0.16 & 0.61 \\
\hline 213 & $21.77 \pm 0.02$ & $20.53 \pm 0.02$ & $1.23 \pm 0.03$ & 0.42 & 1.44 \\
\hline
\end{tabular}

Notes. The sources in the top section of the table, denoted by the horizontal line, have been confirmed as globular clusters, while those in the bottom section of the table have all been identified as background objects.

indicated by the labeling in the panel. In the bottom panel, the error bars for each of these points are plotted. Also in this figure source variability is indicated, where variable sources are plotted in blue, nonvariable sources are shown in green, and sources with undetermined variability are indicated in cyan. Additionally, in both of the panels a grid has been overlaid to indicate the predicted locations of the sources at redshift $z=0$ for different spectra, described by a power law with various photon indices $\left(0 \leqslant \Gamma_{\mathrm{ph}} \leqslant 4\right.$, from top to bottom.) and absorption column densities $\left(10^{20} \leqslant N_{\mathrm{H}} \leqslant 10^{22} \mathrm{~cm}^{-2}\right.$, from right to left). In Figure 12 the $L_{X}-\mathrm{HR}, L_{X}-\mathrm{C} 21$, and $L_{X}-\mathrm{C} 32$ population plots are presented, where variability is again indicated by color, with variable sources shown in blue, nonvariable sources are plotted in green, and sources with undetermined variability are shown in cyan.

From the color-color diagram, presented in Figure 11, it can be seen that most of the well-defined colors lie within the area of a typical LMXB spectrum of $\Gamma=1.5-2.0$, with no intrinsic absorption (e.g., Irwin et al. 2003; Fabbiano 2006). However, there also appears to be a population of sources that have much harder spectra, again with either no intrinsic absorption, or sources with a possible soft excess, albeit with colors that are not as well defined. The $L_{X}-\mathrm{HR}$ distribution of this subpopulation was explored to identify sources with higher 
Table 12

Properties of Optical Sources that have been Classified as "Excluded Matches": Sources Detected Between 0."6 and 3" of an X-Ray Point Source

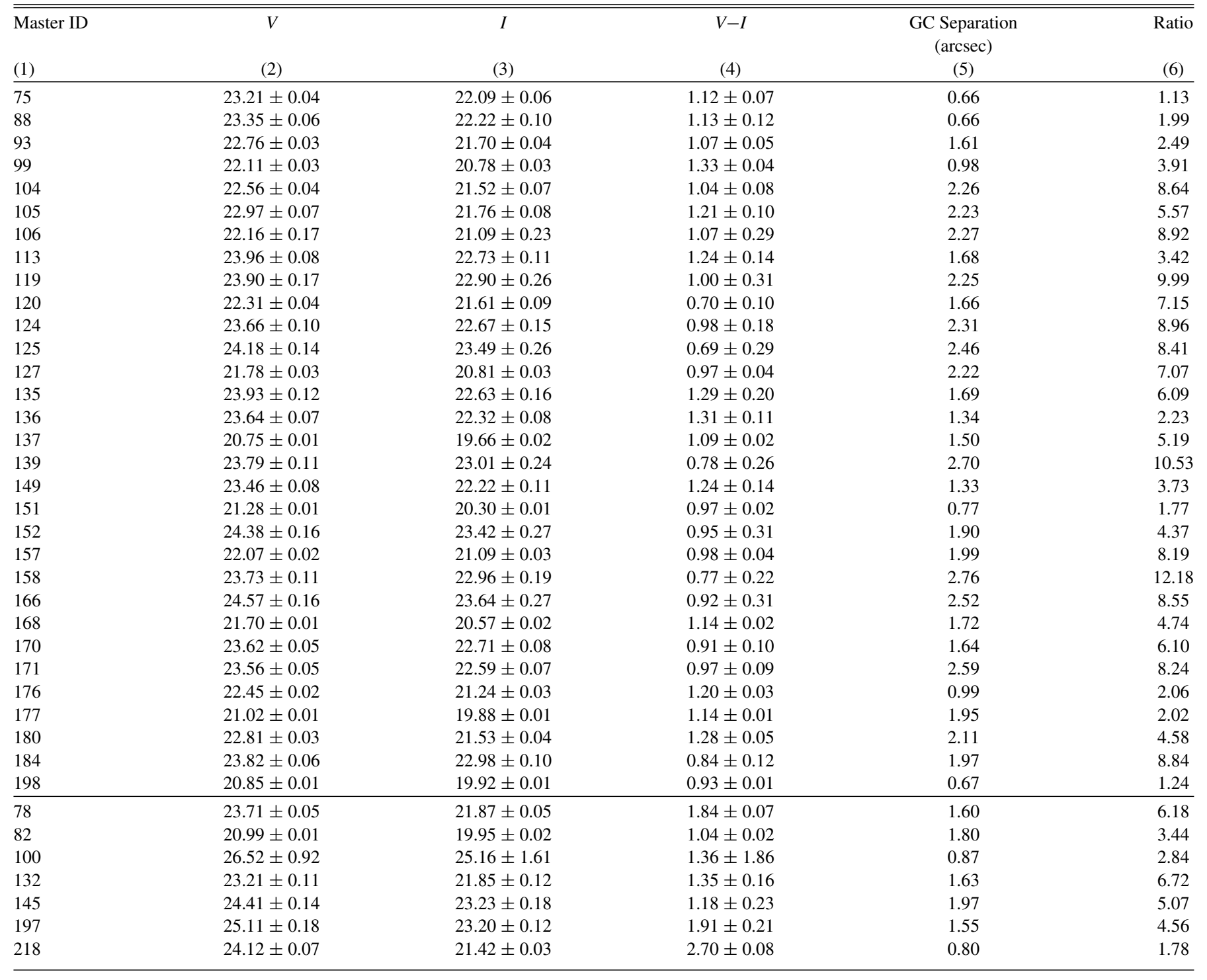

Notes. The sources in the top section of the table, denoted by the horizontal line, have been confirmed as globular clusters, while those in the bottom section of the table have all been identified as background objects.

hardness ratios than one would expect from LMXBs but it was found that the sources that were identified in the color-color diagram did not exhibit harder HR values (see the top panel of Figure 12). Because of this, while it is possible that these sources could be absorbed background active galactic nucleus (AGN), the poorly constrained colors of this subpopulation alone do not provide adequate information to confirm this interpretation. The overall color-color and $L_{\mathrm{X}}-\mathrm{HR}$ distributions of the LMXB population within NGC 4278 is very similar to that of NGC 3379, where again most of the sources with well-constrained colors were determined to lie within the area of a typical LMXB spectrum. Also in NGC 3379, like NGC 4278 , a subpopulation of sources with harder spectra with either no intrinsic absorption, or a soft excess, was identified. However, unlike the subpopulation of NGC 4278, these sources were determined to show not only color-color values indicating their harder spectra, but also exhibited higher HR values and for this reason were flagged as possible absorbed background AGN.

\subsection{Source Variability}

A characteristic of compact accretion sources such as LMXBs is variability, and, as a result of the monitoring nature of the observing campaign, we have been able to search for this variability, in both the long-term regime and also over shortterm baselines, where changes over hours and days have been identified. One of the specific aims of our monitoring campaign has been to identify TC sources as it has been suggested that field LMXBs are expected to be transients (Piro \& Bildsten 2002; King 2002) and low-luminosity ultracompact binaries in GCs are also expected to be transient in nature (Bildsten \& Deloye 2004). In the forthcoming paper N. J. Brassington et al. (2009, in preparation), we investigate the subpopulation of TCs that has been discovered in both NGC 4278 and NGC 3379.

Our data represent the most complete variability study for an extragalactic LMXB population (see Fabbiano 2006; Xu et al. 2005), investigating both long- and short-term behavior. In the 

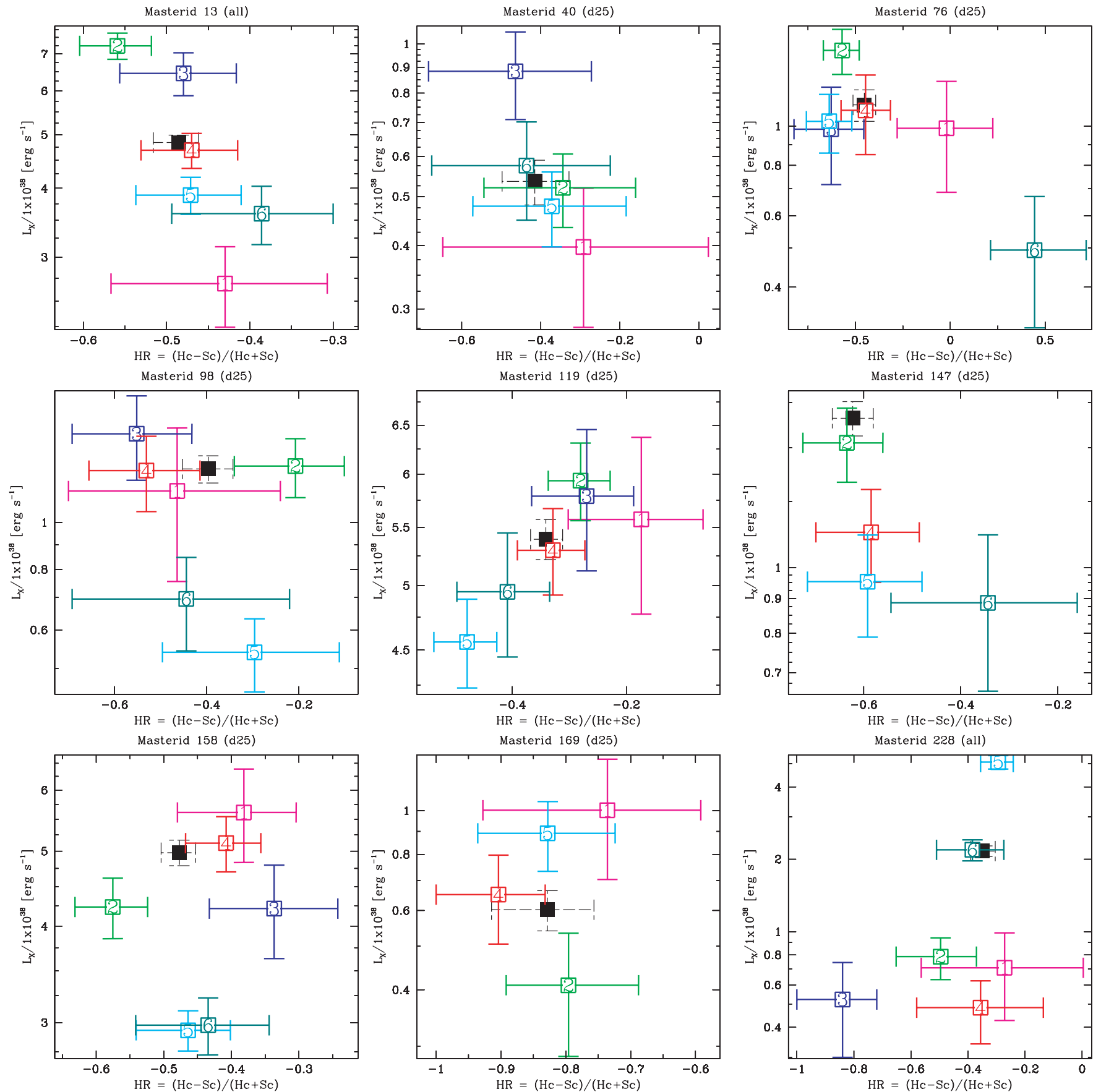

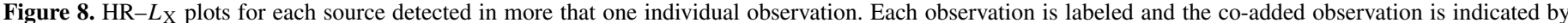

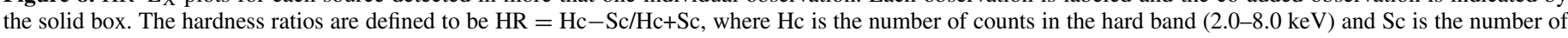
counts in the soft band (0.5-2.0 keV).

(An extended and color version of this figure is available in the online journal.)

case of the long-term variability, sources have been separated into four different classifications: nonvariable and variable sources, and also TCs or PTCs. These two latter definitions have been applied to sources that either appear or disappear, or are only detected for a limited amount of "contiguous" time during the observations, with a lower bound ratio of greater than 10 between the "on-state" and the "off-state," for TCs, or a lower bound ratio between 5 and 10 for the PTCs (see Section 2.4 for a full discussion of this definition).

The 13 sources that were investigated for transient behavior are presented in Table 13, where both the ratio and lower bound ratio, calculated from Bayesian modeling, are presented, along with each source's variability classification. From this table it can be seen that many of these sources appear to be PTCs and TCs from their ratio alone, but when allowing for the uncertainties from their source and background counts, they can only be classified as variable sources. Including the uncertainties when determining TCs is particularly important when dealing with sources with low-S/ $\mathrm{N}$ values, as is the case for a number of sources in this catalog.

Out of the 236 sources, $97,41 \%$ of the sources within NGC 4278 , have been defined as variable sources. A further three sources are TCs, and three are PTCs, with 97 sources found to be 

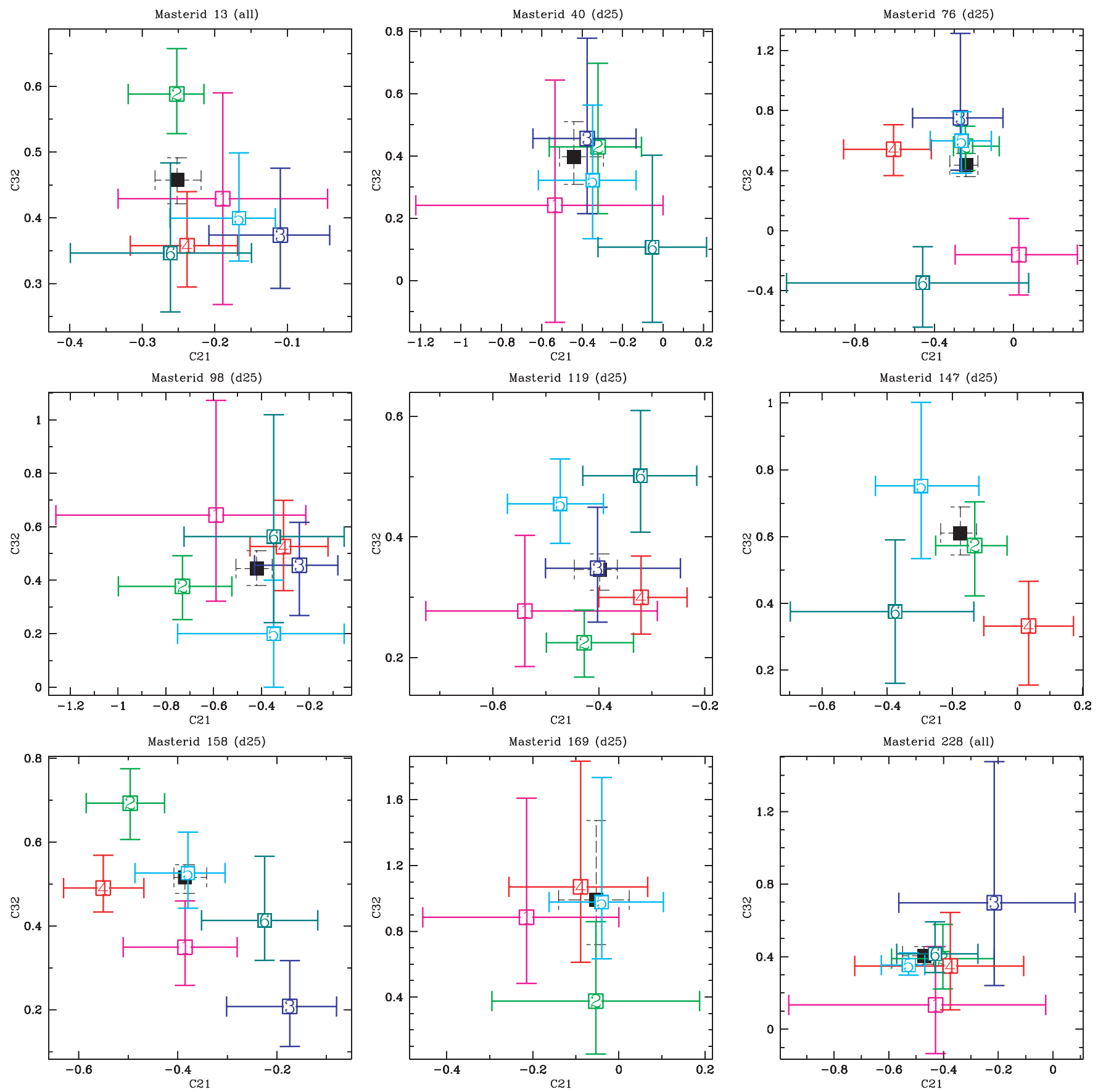

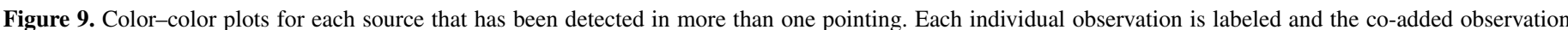

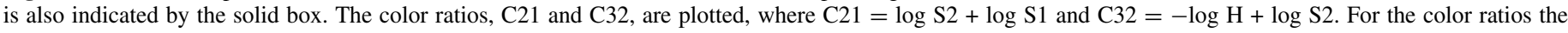
bandwidths are defined to be $\mathrm{S} 1=0.3-0.9 \mathrm{keV}, \mathrm{S} 2=0.9-2.5 \mathrm{keV}$, and $\mathrm{H}=2.5-8.0 \mathrm{keV}$.

(An extended and color version of this figure is available in the online journal.)

nonvarying in intensity over the six observations. The remaining 36 sources have insufficient data to investigate their long-term variability. These levels of long-term variability observed in NGC 4278 are very similar to those of NGC 3379 , where $42 \%$ of the sources were determined to be variable, with a further five sources identified as TCs and three as PTCs. This similar number of TCs/PTCs in both galaxies is, maybe, somewhat surprising, given the larger number of LMXBs detected within NGC 4278. However, from theory it has been suggested that transient sources will predominantly be field LMXBs (Piro \& Bildsten 2002), and in fact in NGC 4278 five of the six TC/ PTC sources have been determined to be field sources and in
NGC 3379 three of the four TC/PTCs that we have optical coverage for are also field sources (the remaining four TC/ PTCs are external to the HST FOV). Both NGC 3379 and NGC 4278 were selected for this study as they have very similar $L_{\mathrm{B}}$ values $\left(1.35 \times 10^{10} L_{\odot}, 1.63 \times 10^{10} L_{\odot}\right)$, and by comparing the number of field TCs/PTCs in each galaxy to their field population we find similar values of $11 \%$ and $9 \%$, respectively, compared to $8 \%$ and $3 \%$ when including the whole $\mathrm{X}$-ray source population within the $D_{25}$ ellipse. These values are consistent with the suggestion that transient sources will be predominantly found in the field, and this result will be further explored in the forthcoming paper N. J. Brassington et al. (2009, in preparation). 
Table 13

Ratio Values of Potential Transient Candidates

\begin{tabular}{lccc}
\hline \hline Master ID & Mode Ratio & Lower Bound Ratio & Variability \\
\hline 27 & 5.0 & 3.6 & $\mathrm{~V}$ \\
30 & 3.2 & 2.2 & $\mathrm{~V}$ \\
32 & 4.5 & 3.7 & $\mathrm{~V}$ \\
59 & 4.8 & 3.5 & $\mathrm{~V}$ \\
83 & 57.8 & 35.5 & $\mathrm{TC}$ \\
91 & 9.0 & 8.3 & PTC \\
92 & 5.8 & 4.3 & $\mathrm{~V}$ \\
113 & 3.5 & 2.4 & $\mathrm{~V}$ \\
139 & 26.1 & 18.9 & $\mathrm{TC}$ \\
152 & 5.3 & 4.2 & $\mathrm{~V}$ \\
156 & 6.2 & 5.5 & PTC \\
159 & 13.6 & 5.8 & PTC \\
204 & 871.7 & 202.9 & TC \\
\hline
\end{tabular}

Notes. The 13 sources that were identified as potential transient candidates. Mode ratios and lower bound ratios were derived from Bayesian modeling, see Section 2.4 for more details. Sources were determined to be transient candidates (TC) if the lower bound $>10$, possible transient candidates (PTC) if the lower bound $>5$, and variable $(\mathrm{V})$ if lower than 5 .

Table 14

Summary of Long-Term and Short-Term Source Variability

\begin{tabular}{lcccccc}
\hline \hline \multirow{2}{*}{$\begin{array}{l}\text { Short-Term } \\
\text { Variability }\end{array}$} & $\mathrm{N}$ & $\mathrm{V}$ & $\mathrm{TC}$ & $\mathrm{PTC}$ & $\mathrm{U}$ & \multirow{2}{*}{ Total } \\
\hline $\mathrm{N}$ & $7 \mathbf{( 7 )}$ & $11(\mathbf{9})$ & $0(\mathbf{0})$ & $0(\mathbf{0})$ & $0(\mathbf{0})$ & $18(\mathbf{1 6})$ \\
$\mathrm{V}$ & $3 \mathbf{( 2 )}$ & $6(\mathbf{5})$ & $0(\mathbf{0})$ & $0(\mathbf{0})$ & $0(\mathbf{0})$ & $9(\mathbf{7})$ \\
$\mathrm{P}$ & $11(\mathbf{7})$ & $14(\mathbf{1 0})$ & $0(\mathbf{0})$ & $0(\mathbf{0})$ & $0(\mathbf{0})$ & $25(\mathbf{1 7})$ \\
$\mathrm{U}$ & $76(\mathbf{5 4})$ & $66(\mathbf{5 2})$ & $3(\mathbf{3})$ & $3(\mathbf{3})$ & $36(\mathbf{2 8})$ & $184(\mathbf{1 4 0})$ \\
Total & $97(\mathbf{7 0})$ & $97(\mathbf{7 6})$ & $3 \mathbf{( 3 )}$ & $3(\mathbf{3})$ & $36(\mathbf{2 8})$ & $236(\mathbf{1 8 0})$ \\
\hline
\end{tabular}

Notes. The long-term variability definitions: $\mathrm{N}$ indicates nonvariable, $\mathrm{V}$ variable, TC transient candidate, PTC possible transient candidate, and U unable to determine variability as all individual observations were upper limits. Shortterm variability definitions: $\mathrm{N}$ indicates nonvariable in all six observations, $\mathrm{V}$ variable in a least one observation, $\mathrm{P}$ possible variability in at least one observation (see Section 2.4 for full definition), and $U$ too few counts in all six observations to determine variability. The bold values in brackets indicate the number of sources within the $D_{25}$ ellipse.
The long- and short-term variability of the X-ray sources with NGC 4278 are summarized in Table 14, where these two variability parameters have been cross-correlated, to indicate the number of sources exhibiting both long- and short-term variations, although the majority of these sources do not have sufficient counts in each observation to determine their shortterm variability. The numbers within this table indicate the number of sources from the whole observation and the numbers in brackets represent the sources within the $D_{25}$ ellipse. From this table it can be seen that, for the sources with a defined short-term variability measure, both long-term variable and nonvariable sources have a variety of short-term behavior. For the TCs no observations had sufficient counts with which to investigate their short-term variability. Also, as an additional point, all of the TCs and PTCs found within NGC 4278 reside well within the $D_{25}$ ellipse of the galaxy, indicating that they are likely LMXBs associated with NGC 4278.

In addition to the $L_{X}$ variability, spectral variations have also been investigated. These are presented in Figures 8 and 9 , where $L_{\mathrm{X}}-\mathrm{HR}$ and color-color plots for each source are shown. From these figures it is clear that the majority of sources within NGC 4278 exhibit some sort of variability, with a variety of different spectral variations shown within this population. This spectral variability is similar to behavior discussed in McClintock \& Remillard (2006), with a significant number of sources emitting softer spectra as $L_{X}$ increases (e.g., sources 76 and 147). It has been suggested that this increasing softness represents a thermal state, where the flux is dominated by the heat radiation from the inner accretion disk. Conversely, sources exhibiting spectral hardening with increasing $L_{\mathrm{X}}$ are also present within the galaxy (e.g., sources 14 and 119). This harder state has been explained as a steep power-law state, where a highly energized corona is present (e.g., Feng \& Kaaret 2006). In addition to these spectral behaviors, sources that show little to no spectral variation with increasing luminosity (e.g., sources 40 and 169) and sources that show no discernible pattern at all (e.g., sources 118 and 158) have also been detected. A more detailed discussion of the spectral variability of all of these X-ray sources presented in this catalog will be the subject of a forthcoming paper.

Table 15

Raw Source and Background Counts from the Co-Added Observation

\begin{tabular}{|c|c|c|c|c|c|c|c|c|c|c|c|c|c|c|c|}
\hline \multirow[t]{2}{*}{ Master ID } & \multirow[t]{2}{*}{ Net $B$ Counts } & \multicolumn{2}{|c|}{ B-Band } & \multicolumn{2}{|c|}{ S1-Band } & \multicolumn{2}{|c|}{ S2-Band } & \multicolumn{2}{|c|}{$\begin{array}{c}\text { Raw Counts } \\
\text { H-Band }\end{array}$} & \multicolumn{2}{|c|}{ Bc-Band } & \multicolumn{2}{|c|}{ Sc-Band } & \multicolumn{2}{|c|}{ Hc-Band } \\
\hline & & $(\mathrm{Src})$ & (Bkg) & $(\mathrm{Src})$ & (Bkg) & $(\mathrm{Src})$ & (Bkg) & $(\mathrm{Src})$ & (Bkg) & $(\mathrm{Src})$ & (Bkg) & $(\mathrm{Src})$ & (Bkg) & $(\mathrm{Src})$ & (Bkg \\
\hline (1) & (2) & (3) & (4) & (5) & (6) & (7) & (8) & (9) & (10) & (11) & (12) & (13) & (14) & (15) & (16) \\
\hline 1 & 139.8 & 198.0 & 58.2 & 13.0 & 15.3 & 61.0 & 13.0 & 124.0 & 29.8 & 191.0 & 48.5 & 54.0 & 14.9 & 137.0 & 33.6 \\
\hline 2 & 386.2 & 458.0 & 71.3 & 74.0 & 16.5 & 254.0 & 16.6 & 130.0 & 38.2 & 438.0 & 61.9 & 278.0 & 19.4 & 160.0 & 42.5 \\
\hline 3 & 7.8 & 69.0 & 61.9 & 15.0 & 15 . & 25.0 & 15.5 & 29.0 & 30.6 & 61.0 & 53.1 & 29.0 & 18.7 & 32.0 & 34.3 \\
\hline 4 & 266.1 & 333.0 & 66.6 & 94.0 & 15.2 & 162.0 & 16.7 & 77.0 & 34.8 & 312.0 & 58.1 & 215.0 & 19.3 & 97.0 & 38.9 \\
\hline 5 & 725.7 & 787.0 & 61.3 & 217.0 & 14.8 & 413.0 & 14.7 & 157.0 & 31.8 & 737.0 & 53.6 & 527.0 & 18.4 & 210.0 & 35.3 \\
\hline 6 & 17.5 & 75.0 & 58.1 & 9.0 & 15.7 & 23.0 & 13.9 & 43.0 & 28.6 & 72.0 & 49.4 & 22.0 & 17.3 & 50.0 & 32.1 \\
\hline 7 & $\ldots$ & $\ldots$ & $\ldots$ & $\ldots$ & $\ldots$ & $\ldots$ & $\ldots$ & & $\ldots$ & $\ldots$ & & & & & \\
\hline 8 & 128.6 & 180.0 & 51.4 & 44.0 & 11.1 & 89.0 & 12.4 & 47.0 & 28.0 & 169.0 & 45.0 & 108.0 & 14.2 & 61.0 & 30.9 \\
\hline 9 & 46.7 & 108.0 & 61.3 & 31.0 & 14.6 & 39.0 & 15.4 & 38.0 & 31.3 & 97.0 & 52.7 & 52.0 & 17.1 & 45.0 & 35.6 \\
\hline 10 & 7.7 & 44.0 & 36.3 & 13.0 & 8.1 & 15.0 & 9.9 & 16.0 & 18.4 & 39.0 & 32.4 & 21.0 & 11.6 & 18.0 & 20.7 \\
\hline
\end{tabular}

Notes. Column 1: master ID; Column 2: net broadband counts. Columns 3, 5, 7, 9, 11, 13, and 15: raw source counts in each of the seven energy bands (see Table 2 for definitions of these bands); Columns 4, 6, 8, 10,12,14, and 16: background counts in each of the seven energy bands. In some instances background counts are very low. For these sources standard aperture photometry results in negative net counts, so, instead, the source cell determined by wavdetect has been used. This results in a large area ratio between the background and source regions and therefore a low background count value is derived.

(This table is available in its entirety in a machine-readable form in the online journal. A portion is shown here for guidance regarding its form and content.) 

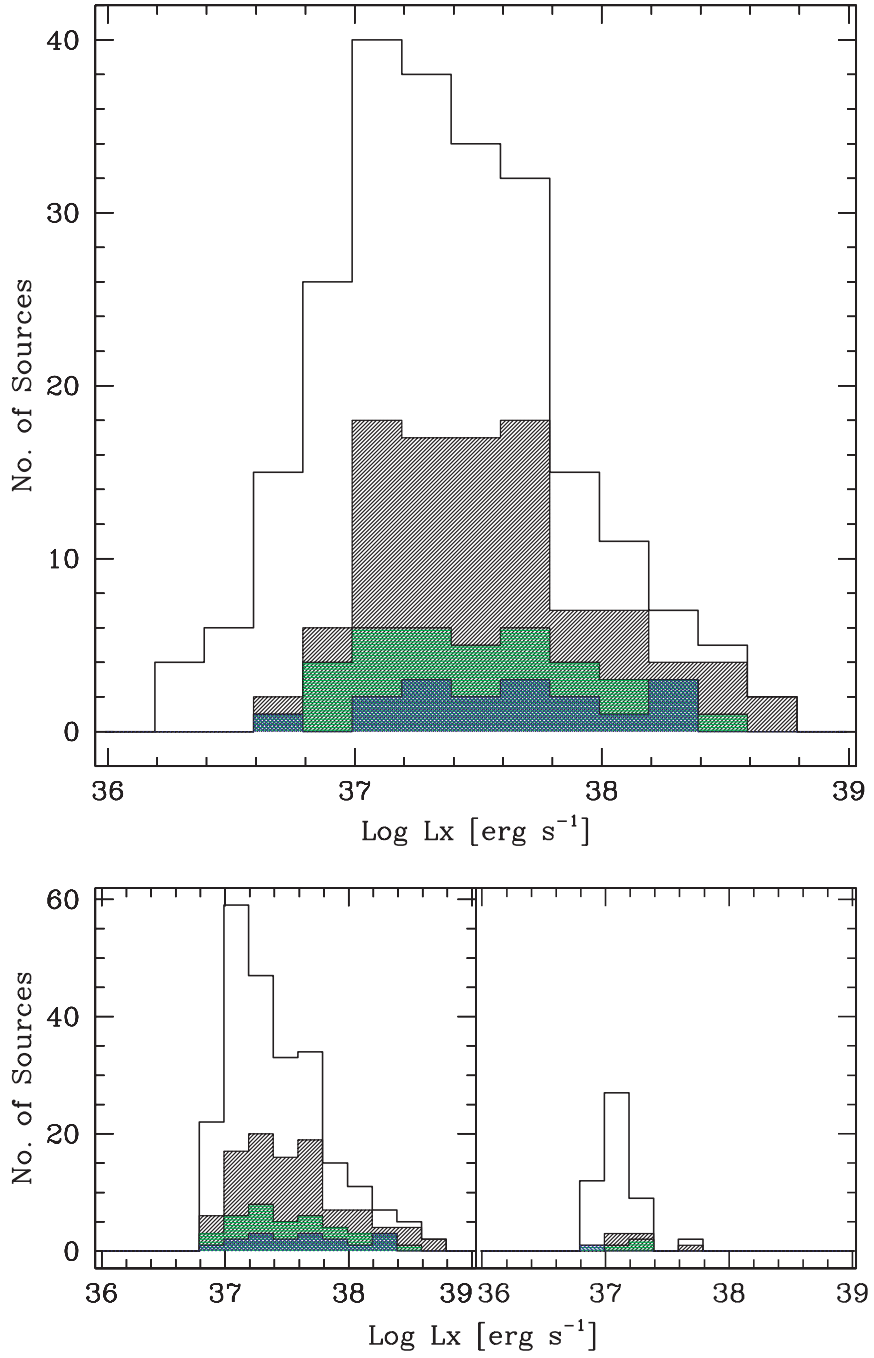

Figure 10. Top figure presents the $L_{\mathrm{X}}$ distribution of the 236 sources detected within the overlapping region, covered by all six Chandra pointings. The unshaded histogram indicates all detected sources. The lightly shaded (gray) region shows all variable sources (including both transient classes). The darker (green) histogram indicates sources associated with a GC and the darkest (blue) histogram shows varying sources that have a confirmed GC counterpart. The bottom left image indicates the same 236 sources, but for those with $\mathrm{S} / \mathrm{N}<3$, $3 \sigma$ upper limit values have been used in place of $L_{\mathrm{X}}$. The bottom right image presents these upper limit values only. The shading for these two figures are the same as described for the main histogram.

\section{CONCLUSIONS}

We have presented a source catalog and variability atlas resulting from our monitoring deep observations of the nearby elliptical NGC 4278 with Chandra ACIS-S. Our results can be summarized as follows.

1. $236 \mathrm{X}$-ray point sources have been detected within NGC 4278 , ranging in luminosity from $3.5 \times 10^{36} \mathrm{erg} \mathrm{s}^{-1}$ (with $3 \sigma$ upper limit $\leqslant 1 \times 10^{37} \mathrm{erg} \mathrm{s}^{-1}$ ) to $\sim 2 \times 10^{40} \mathrm{erg} \mathrm{s}^{-1}$, with 180 of these sources residing within the $D_{25}$ ellipse of the galaxy.

2. The nuclear source in this galaxy has been classified as a LINER with $\mathrm{H} \alpha$ emission (Ho et al. 1997) and has a luminosity of $\sim 2 \times 10^{40} \mathrm{erg} \mathrm{s}^{-1}$ from the co-added observation.

3. 39 GCs have been identified to be coincident with Xray sources, all of which lie within the $D_{25}$ ellipse of the galaxy. These GC-LMXB associations tend to have high
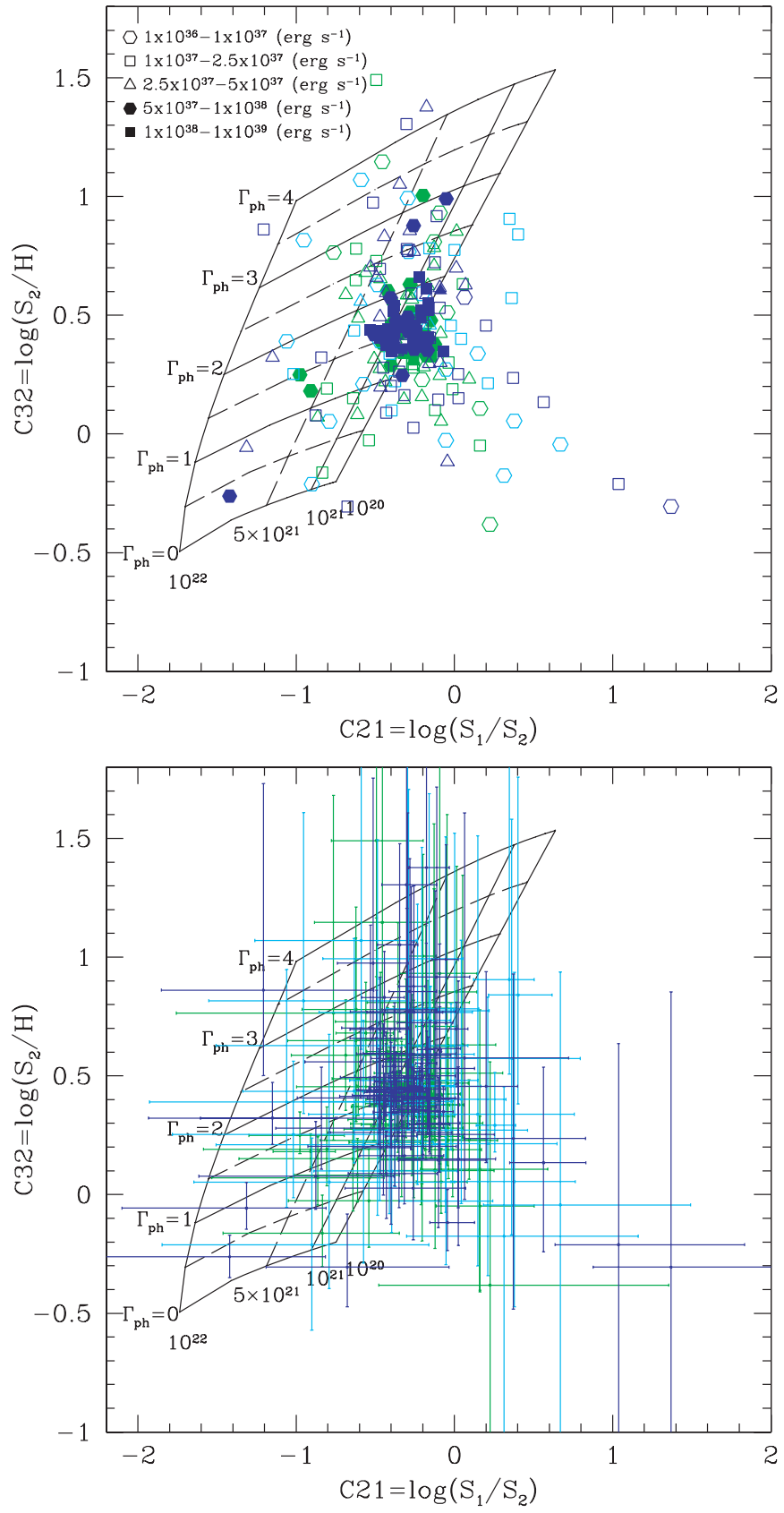

Figure 11. Color-color diagram of the X-ray point sources detected in the co-added observation. In the top panel color-color values are plotted, with the sources divided into luminosity bins, with symbols of each bin indicated by the labeling in the panel. Variability is also indicated, where variable sources are shown in blue, nonvariable source are indicated in green, and source that have insufficient counts to identify variability are shown in cyan. In the lower panel, the error values for each of the sources are presented. In both of the panels, the grid indicates the predicted locations of the sources at redshift $z=0$ with various photon indices $\left(0 \leqslant \Gamma_{\mathrm{ph}} \leqslant 4\right.$, from top to bottom) and absorption column densities $\left(10^{20} \leqslant N_{\mathrm{H}} \leqslant 10^{22} \mathrm{~cm}^{-2}\right.$, from right to left).

X-ray luminosities, with 10 of these sources exhibiting $L_{\mathrm{X}}>1 \times 10^{38} \mathrm{erg} \mathrm{s}^{-1}$.

4. From source photometry, it has been determined that the majority of source with well-constrained colors have values that are consistent with a typical LMXB spectrum of $\Gamma=1.5-2.0$, with no intrinsic absorption.

5. 103 sources, $44 \%$ of the X-ray source population, have been found to exhibit some type of long-term variability, which clearly identifies them as accreting compact objects. Three 

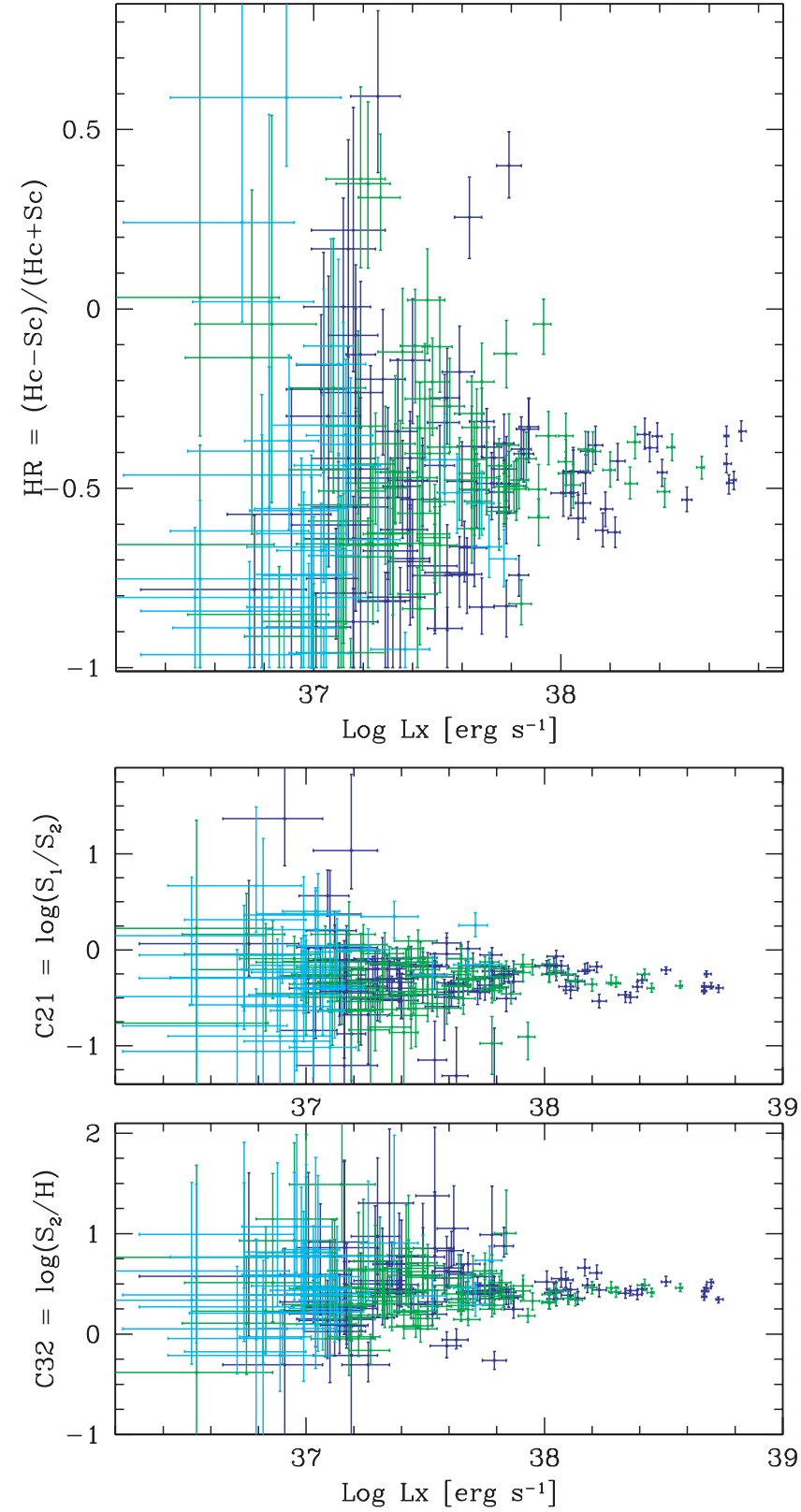

Figure 12. Top panel presents the $L_{X}-H R$ diagram of the $X$-ray point sources detected in the co-added observation. The second panel shows the $L_{X}-\mathrm{C} 21$ plot for this population and the bottom panel shows the $L_{X}-\mathrm{C} 32$ values. In all three panels the variable sources are plotted in blue, nonvariable sources in green, and the sources without determined variability are plotted in cyan.

of these variable sources have been identified as TCs, with a further three identified as possible transients.

6. Spectral variability analysis has revealed that the sources within NGC 4278 exhibit a range of variability patterns, where both high/soft-low/hard and low/soft-high/hard spectral transitions have been observed, as well as sources that vary in luminosity, but exhibit no spectral variation, indicating that there are many different source classes within this galaxy.

Following this catalog paper, we will be presenting highlights from the X-ray binary population of this galaxy in forthcoming papers. The first compares the XLF of GC and field LMXBs detected in both NGC 4278 and NGC 3379 (Kim et al. 2009). This will be followed by a paper presenting the properties of the transient population of both galaxies (N. J. Brassington et al. 2009 , in preparation). In addition to these we will also present papers on the properties of the nuclear source and the diffuse emission of NGC 4278, as well as the nonuniform distribution of the sources within the galaxy and the intensity and spectral variability of the luminous X-ray binary population.

We thank the CXC DS and SDS teams for their efforts in reducing the data and developing the software used for the reduction (SDP) and analysis (CIAO). We also thank the referee, Jimmy Irwin, for his helpful comments which have improved this paper. This work was supported by Chandra G0 grant G06-7079A (PI:Fabbiano) and subcontract G067079B (PI: Kalogera). We acknowledge partial support from NASA contract NAS8-39073 (CXC). A. Zezas acknowledges support from NASA LTSA grant NAG5-13056. S. Pellegrini acknowledges partial financial support from the Italian Space Agency ASI (Agenzia Spaziale Italiana) through grant ASIINAF I/023/05/0.

\section{REFERENCES}

Bildsten, L., \& Deloye, C. J. 2004, ApJ, 607, L119

Brassington, N. J., et al. 2008, ApJS, 179, 142

Cappellari, M., et al. 2006, MNRAS, 366, 1126

Fabbiano, G. 2006, ARAA, 44, 323

Feng, H., \& Kaaret, P. 2006, ApJ, 650, L75

Freeman, P. E., Kashyap, V., Rosner, R., \& Lamb, D. Q. 2002, ApJS, 138, 185 Gehrels, N. 1986, ApJ, 303, 336

Giacconi, R. 1974, in X-Ray Astronomy, ed. R. Giacconi, \& H. Gursky (Dordrecht: Reidel) 155

Gilfanov, M. 2004, MNRAS, 349, 146

Grindlay, J. E. 1984, Adv. Space Res., 3, 19

Harris, W. E. 1991, ARAA, 29, 543

Ho, L. C., Filippenko, A. V., Sargent, W. L. W., \& Peng, C. Y. 1997, ApJS, 112 39

Irwin, J. 2005, ApJ, 631, 511

Irwin, J. 2006, MNRAS, 371, 1903

Irwin, J. A., Athey, A. E., \& Bregman, J. N. 2003, ApJ, 587, 356

Irwin, J. A., Bregman, J. N., \& Athey, A. E. 2004, ApJ, 601, L143

Kenter, A., et al. 2005, ApJS, 161, 9

Kim, D.-W., et al. 2004a, ApJS, 150, 19

Kim, D.-W., et al. 2004b, ApJ, 600, 59

Kim, D.-W., et al. 2006, ApJ, 652, 1090

Kim, D.-W., et al. 2009, ApJS, submitted

Kim, M., et al. 2007a, ApJS, 169, 401

Kim, M., et al. 2007b, ApJ, 659, 29

King, A. R. 2002, MNRAS, 335, L13

Kraft, R. P., et al. 2001, ApJ, 560, 675

Kundu, A., \& Whitmore, B. C. 2001, ApJ, 121, 2950

McClintock, J. E., \& Remillard, R. A. 2006, in Compact Stellar X-Ray Sources, ed. W. H. G. Lewin \& M. van der Klis (Cambridge: Cambridge Univ. Press), p. 157

Park, T., Kashyap, V., Siemiginowska, A., van Dyk, D., Zezas, A., Heinke, C., \& Wargelin, B. 2006, ApJ, 652, 610

Piro, A. L., \& Bildsten, L. 2002, ApJ, 571, L103

Scargle, J. D. 1998, ApJ, 504, 405

Sivakoff, G. R., Jordán, A., Juett, A. M., Sarazin, C. L., \& Irwin, J. A. 2008 arXiv:0806.0627

Terlevich, A. I., \& Forbes, D. A. 2002, MNRAS, 330, 547

Tonry, J., et al. 2001, ApJ, 546, 681

van Dyk, D., et al. 2001, ApJ, 548, 224

Verbunt, F., \& van den Heuvel, E. P. J. 1995, in X-Ray Binaries, ed. W. H. G. Lewin, J. van Paradijs, \& E. P. J. van den Heuvel (Cambridge: Cambridge Univ. Press) 457

Voss, R., \& Gilfanov, M. 2006, A\&A, 447, 71

Weisskopf, M. C., Tananbaum, H. D., Van Speybroeck, L. P., \& O’Dell, S. L. 2000, Proc. SPIE, 4012, 2

Williams, B. F., et al. 2008, ApJ, 680, 1120

Xu, Y., Xu, H., Zhang, Z., Kundu, A., Wang, Y., \& Wu, X.-P. 2005, ApJ, 631, 809

Zezas, A, Fabbiano, G., Rots, A. H., \& Murray, S. S. 2002, ApJ, 577, 710 